\title{
Complex resonances and trapped modes in ducted domains
}

\author{
By YUTING DUA N ${ }^{1}$, WERNER KOCH , $^{2}$ \\ CHRIS M. LINTON AND MAUREEN MCIVER \\ ${ }^{1}$ Agat Labs Ltd, Calgary, Canada \\ ${ }^{2}$ Institute of Aerodynamics and Flow Technology, DLR Göttingen, Germany \\ ${ }^{3}$ Department of Mathematical Sciences, Loughborough University, Leics, UK
}

(Received 15 February 2006)

\begin{abstract}
Due to radiation losses, resonances in open systems are generally complex valued. However, near symmetric, centred objects in ducted domains, or in periodic arrays, so-called trapped modes can exist below the cut-off frequency of the first non-trivial duct mode. These trapped modes have no radiation loss and correspond to real-valued resonances. Above the first cut-off frequency isolated trapped modes exist only for specific parameter combinations. These isolated trapped modes are termed embedded, because their corresponding eigenvalues are embedded in the continuous spectrum of an appropriate differential operator. Trapped modes are of considerable importance in applications because at these parameters the system can be excited easily by external forcing. In the present paper directly computed embedded trapped modes are compared with numerically obtained resonances for several model configurations. Acoustic resonances are also computed in two-dimensional models of a butterfly and ball-type valve as examples of more complicated geometries.
\end{abstract}

\section{Introduction}

In contrast to completely open systems where all modes are leaky, i.e. have radiation losses, hard-walled obstacles or bodies with a different index of refraction in ducted domains (or laterally periodic arrays) may sustain so-called trapped modes. These trapped modes are confined to the vicinity of the obstacle and do not radiate energy, i.e. nominally have no radiation loss. Therefore, they are of great physical importance because at the trapped-mode frequencies the response to forced excitation can be quite high. Mathematically trapped modes are also of considerable interest because they imply nonuniqueness in the associated scattering problem. Trapped modes correspond to real eigenvalues of the relevant operator in an unbounded domain and are usually associated with a cut-off phenomenon. It is well known that in waveguides a finite number of eigenfunctions, so-called cut-on modes, propagate undamped along the waveguide while all other so-called cut-off modes decay. In waveguide-like environments only the cut-on modes can radiate energy to infinity. At the cut-on condition the eigenfunction of this particular mode is a standing wave between the opposing walls and does not propagate along the waveguide. The existence of trapped modes was first established in the theory of water waves, cf. Ursell (1951). Trapped modes can be observed in various physical contexts including electromagnetic, elastic, acoustic and water waves. Here we limit our- 
selves to acoustic waves about hard-walled obstacles in two-dimensional ducts. Almost identical formulations can be given for water waves in a channel.

If the obstacle is symmetric and positioned symmetrically about the waveguide centreline the solution can be decomposed into a symmetric and antisymmetric part about that line. The continuous spectrum of the antisymmetric solution has a non-zero lower limit given by the first duct cut-off frequency, and the corresponding antisymmetric trapped modes can be found as discrete eigenvalues below the first cut-off frequency as demonstrated by Evans \& Linton (1991). For symmetric obstacles Evans et al. (1994) proved the existence of trapped modes for fairly general geometries and a substantial bibliography is devoted to the numerical study of trapped modes. The first experimental observation of acoustic trapped modes was made by Parker (1966), see also the survey paper by Parker \& Stoneman (1989). Parker (1966) investigated cascades of flat plates in a wind tunnel and found that the vortices shed from the trailing edge can excite purely acoustic resonances with high-amplitude tonal noise, the amplitude of which is limited only by nonlinearity. Using a numerical relaxation technique Parker (1967) was able to compute the resonant frequencies of these trapped modes and found good agreement with his experimental results. Subsequently the finite-length rigid plate on the centreline aligned with the duct walls became the model problem for trapped modes about slender obstacles and various methods were applied to compute these so-called Parker modes: Franklin (1972) used a variational formulation, Nayfeh \& Huddleston (1979), Evans \& Linton (1994) and Duan (2004) applied the mode matching method, and Koch (1983), Evans \& Linton (1991) and Woodley \& Peake (1999) employed the Wiener-Hopf technique. Very similar problems occur in quantum waveguides, where the trapped modes are known as bound states, cf. Exner et al. (1996) or Linton \& Ratcliffe (2004) (see also Grikurov (2004)).

For thick obstacles the single circular cylinder placed on the centreline of an acoustical waveguide became a widely used model problem. Callan et al. (1991) established the existence of trapped modes for sufficiently small cylinders using multipole expansions. Evans \& Porter (1997) extended the solution to the case of any number of circular cylinders of arbitrary size, all positioned on the centreline of the duct, cf. also Maniar \& Newman (1997). Trapped modes were also found by Utsunomiya \& Taylor (1999) as well as Porter \& Evans (1999) for a row of cylinders placed perpendicular to the channel walls, and an existence proof was given by Linton \& McIver (2002). All the above theoretical results and solution procedures make use of the special shape of the obstacles and lead to very efficient methods of computation. Recently Hein et al. (2004) introduced a purely numerical method for the computation of both real and complex resonances of abitrarily shaped objects by using Perfectly Matched Layer (PML) absorbing boundary conditions which approximate Sommerfeld's radiation condition at the necessarily finite grid boundaries. This numerical method can not prove the existence of trapped modes but gives a strong indication of their existence if the imaginary part of the resonant frequency is vanishingly small, i.e. the radiation loss tends to zero within numerical accuracy. Furthermore, this method can be used for the computation of complex resonances about arbitrary obstacles which are open on all sides, cf. Hein et al. (2005).

A mathematically more complicated situation arises for the computation of trapped modes if the obstacle is no longer symmetric about the waveguide centreline, or for a symmetric obstacle if the frequency is above the first duct cut-off frequency. In this case possible trapped mode frequencies are discrete and embedded in the continuous spectrum of the relevant operator and one speaks of embedded trapped modes, cf. Evans et al. (1993). Aslanyan et al. (2000) showed that if the symmetry is broken the real eigenvalues transform into complex resonances of so-called leaky modes with radiation 
losses. On the other hand Evans et al. (1993) proved the existence of trapped modes for an off-center plate of finite length placed parallel to the duct walls, showing that symmetry is not a necessary requirement for the existence of trapped modes. Following ideas of Davies \& Parnovski (1998) and Groves (1998) Linton et al. (2002) considered the nonsymmetrical obstacle as part of an infinite array of obstacles symmetric about the sound-hard duct wall (Neumann problem) or antisymmetric about the soft duct wall (Dirichlet problem) and were able to compute embedded trapped modes above the first cut-off frequency. Recently Porter \& Evans (2005) demonstrated for periodic arrays of obstacles with rectangular cross-section the existence of embedded trapped Rayleigh-Bloch modes above the first cut-off frequency of the array. The body of work just described suggests that trapped modes exist in all frequency ranges, but for each additional propagating cut-on mode that is introduced an extra geometrical parameter is required to satisfy side conditions which force the amplitude of these cut-on modes to zero.

The first numerical evidence for the existence of an isolated embedded trapped mode above the first cut-off frequency for a circular cylinder placed on the centreline of the duct was published by Evans \& Porter (1998). McIver et al. (2001) then showed that this isolated embedded trapped mode above the first cut-off frequency is one of a continuous branch of trapped modes for two-parameter obstacles. One such branch was found for a family of ellipses of varying aspect ratio and size beginning with a trapped mode for a flat plate aligned parallel to the duct wall and ending with a standing wave for a semiinfinite duct closed with a hard wall on one side. In a follow-up paper McIver et al. (2002) showed the existence of further branches of embedded trapped modes for both ellipses and rectangular blocks, each of which start with a plate of different length on the centreline of the duct. Isolated embedded trapped modes are of physical importance because at these specific conditions the system can be excited easily even though the frequency is in the leaky mode regime.

Evans \& Linton (1991) found trapped modes not only near obstacles in a channel but also near indentations in the channel wall. In his PhD thesis Duan (2004) extended this analysis to embedded trapped modes above the first cut-off frequency, with first results published in Duan \& McIver (2002). The numerical resonance computations of Koch (2005) also strongly indicate the existence of embedded trapped modes above the first cut-off frequency for rectangular indentations in a duct. The main objective of the present paper is to combine the two approaches, namely the direct computation of embedded trapped modes and the numerical computation of complex resonances in ducted domains and show that the embedded trapped modes agree exactly with the resonances having a vanishingly small imaginary part.

\section{Problem formulation and solution methods}

The propagation of small acoustic disturbances in an infinitely long duct of uniform height $h^{*}$ with zero mean flow and containing an arbitrary object is governed by the wave equation, the propagation velocity being the ambient speed of sound $c_{0}{ }^{*}$. Here, and in the following, the star superscript marks dimensional quantities. We formulate our problems in two-dimensional Cartesian coordinates $(x, y)$ nondimensionalised with a characteristic reference length $L_{r e f}$ which in all our examples is chosen to be the duct height $h^{*}$ such that $h=h^{*} / L_{r e f}^{*}=1$. All velocities are nondimensionalised with $c_{0}{ }^{*}$,

densities with the ambient density $\rho_{0}{ }^{*}$ and pressures with $\rho_{0}{ }^{*} c_{0}^{* 2}$. Assuming harmonic time dependence $\exp \left(-i \omega^{*} t^{*}\right)$, where $\omega^{*}$ is the circular frequency, the wave equation 

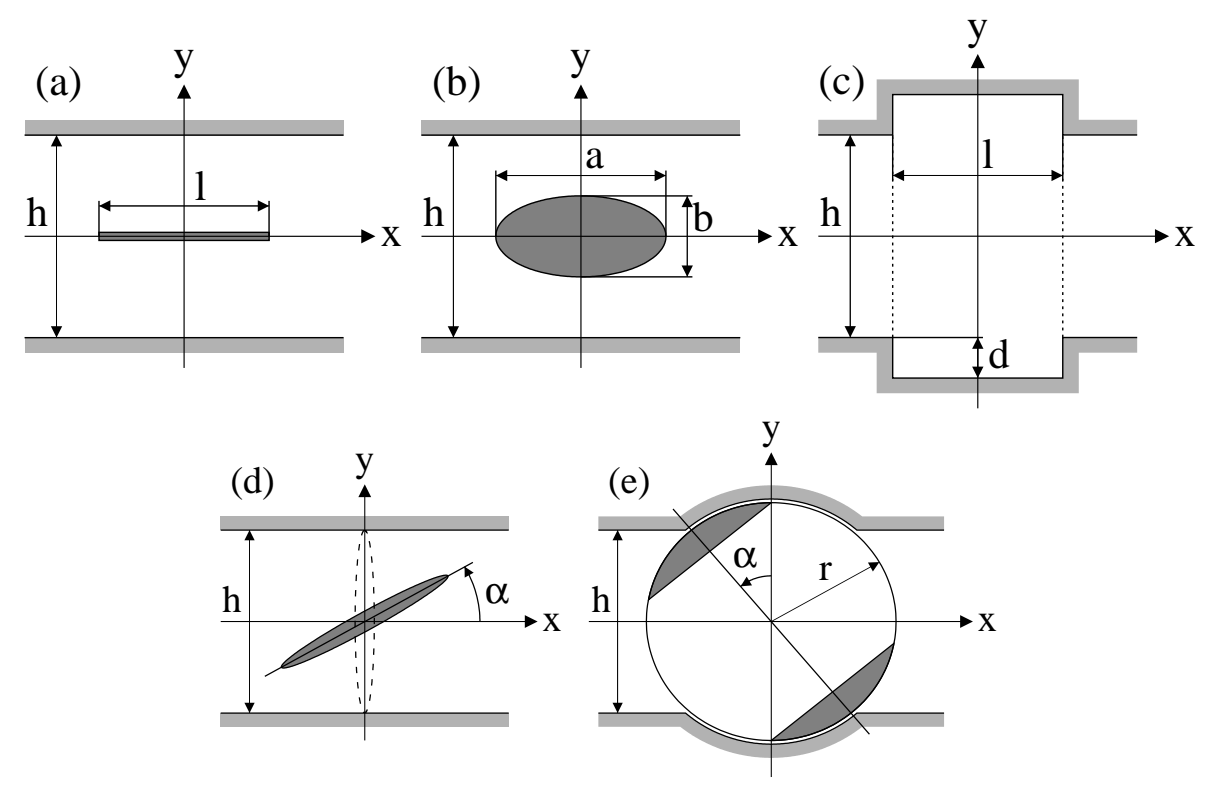

Figure 1. Model configurations: (a) Parker mode problem, (b) centred elliptical cylinder in duct, (c) rectangular indentations in duct wall, (d) model of butterfly valve, (e) model of ball-type valve.

reduces to the nondimensional Helmholtz equation

$$
\left(\Delta+K^{2}\right) \phi=0
$$

for the velocity potential $\phi(x, y)$. The symbol $\Delta=\partial^{2} / \partial x^{2}+\partial^{2} / \partial y^{2}$ is the two-dimensional Laplacian and $K=\omega^{*} L_{r e f}^{*} / c_{0}{ }^{*}$ denotes a dimensionless frequency, with $K / 2 \pi$ being the Helmholtz number. The time-independent dimensionless disturbance velocity and pressure are then given by $\mathbf{v}(x, y)=\nabla \phi$ and $p(x, y)=i K \phi$ respectively.

On hard walls of any object in the duct the Neumann boundary condition $\partial \phi / \partial n=0$ is applied. Furthermore, the Sommerfeld radiation condition for guided waves,

$$
\lim _{|x| \rightarrow \infty}\left(\frac{\partial \phi}{\partial x}-i K \phi\right)=0,
$$

has to be satisfied uniformly at infinity allowing only outgoing waves. On the duct walls we prescribe either Neumann boundary conditions for a hard-walled duct (Neumann problem denoted by $N$ ) or Dirichlet boundary conditions for a soft-walled duct (Dirichlet problem denoted by $D$ ). In the following sections we shall investigate the resonances, trapped modes and embedded trapped modes for the configurations sketched in figure 1, namely:

(i) Finite length plate centred in duct (Parker mode problem), cf. figure 1a.

(ii) Circular or elliptical cylinder centred in duct, cf. figure $1 \mathrm{~b}$.

(iii) Rectangular indentations in duct wall, cf. figure 1c.

(iv) Rotated elliptical cylinder in duct (model for butterfly valve), cf. figure 1d.

(v) Model for ball-type valve, cf. figure $1 \mathrm{e}$.

In the last two examples we compute acoustic resonances for two different models of control valves in an infinitely long two-dimensional duct. Valves are a major source of vibrations and noise in piping systems, cf. Reethof (1978), which might be enhanced by these shear-layer excited acoustic resonances. 


\subsection{Computation of complex resonances}

Resonances in open systems are frequencies at which solutions of the homogeneous equation (2.1) obeying homogeneous boundary conditions and the radiation condition (2.2) can be found. In general energy is radiated to infinity such that these resonances are complex valued. In the following we shall compute the resonances numerically and therefore have to truncate our domain. In order to avoid unphysical reflections at these finite domain boundaries we apply perfectly matched layer (PML) absorbing boundary conditions, introduced by Bérenger (1994), in the form of the older complex scaling method of atomic and molecular physics, cf. the recent overview by Moiseyev (1998), as used in Hein et al. (2004). For our ducted domains one-dimensional PMLs are sufficient and the complex scaling method works as follows: in the PML domain $\phi(x, y)$ is continued analytically with respect to the variable $x$ to the complex variable $\xi$, e.g.

$$
\xi(x)=x+i \sigma(x) .
$$

The spatial damping function $\sigma(x)$ is usually chosen in power form, smoothly starting at the PML interface at $x= \pm x_{P M L}$, e.g.,

$$
\sigma(x)= \begin{cases}\sigma_{0}\left(x-x_{P M L}\right)^{\beta}, & x>x_{P M L}, \\ 0, & |x| \leq x_{P M L}, \\ -\sigma_{0}\left(-x-x_{P M L}\right)^{\beta}, & x<-x_{P M L} .\end{cases}
$$

For a positive damping coefficient $\sigma_{0}$ and a constant shape parameter $\beta \geq 1$ (we chose $\beta=1$ for all our computations, cf. Hein et al. (2004)) outgoing waves will decay exponentially in the PML. One can therefore truncate the PML at $\pm\left(x_{P M L}+d_{P M L}\right)$ where $d_{P M L}$ denotes the width of the PML. The error due to artificial reflections at this truncated outer edge of the PML is small if $\sigma_{0}$ and $d_{P M L}$ are chosen properly. Therefore, a Dirichlet (or Neumann) boundary condition can be imposed at the outer edge of the PML instead of enforcing Sommerfeld's radiation condition (2.2), cf. Collino \& Monk (1998). In this way a finite domain eigenvalue problem results which can be solved numerically by standard codes for large eigenvalue problems. If the computational domain can be subdivided into rectangular subdomains we use the multi-domain Chebyshev collocation code employed in Hein et al. (2004). For more general boundaries we apply the finite-element code NGSolve of Joachim Schöberl together with his grid generation code NETGEN, cf. Schöberl (1997), which we tested in Hein et al. (2005).

The close relation between the PML method of Bérenger (1994) and the complex scaling method of atomic and molecular physics, the so-called Aguilar-Balslev-CombesSimon theory, cf. Aguilar \& Combes (1971), Baslev \& Combes (1971), Simon (1973), becomes apparent if the PML method is considered in the complex coordinate stretching formulation of Chew \& Weedon (1994) or Chew et al. (1997). They used

$$
\xi(x, \omega)=x+i \sigma(x) / \omega
$$

instead of (2.3). For diffraction problems the frequency $\omega$ is real and prescribed such that (2.5) merely correponds to a scaling of $\sigma_{0}$. However, in our resonance problem $\omega$ is complex and unknown and (2.5) leads to a nonlinear eigenvalue problem. By choosing $\sigma_{0}$ appropriately in the complex scaling formulation (2.3) the eigenvalue problem is linear and therefore considerably smaller.

\subsection{Direct computation of embedded trapped modes}

For special geometries a direct computation of embedded trapped modes is possible. Here full details are given of the computation of embedded modes which are symmetric in both $x$ and $y$, in a Neumann guide with a rectangular indentation. This problem 
(denoted by $N S S$ ) allows a mode matching approach. The details for modes which are antisymmetric in $x$ and symmetric in $y$ (denoted by $N A S$ ) or symmetric in $x$ and antisymmetric in $y$ (denoted by $N S A$ ) or antisymmetric in both $x$ and $y$ (denoted by $N A A$ ), are not presented, but follow in a similar fashion.

In the region $0 \leq x \leq l / 2$ the trapped mode potential which is symmetric in both $x$ and $y$ is expressed as the eigenfunction expansion

$$
\phi=\sum_{n=0}^{\infty} \frac{\epsilon_{n} U_{n}^{I} \cosh k_{n} x}{k_{n} \cosh \left(k_{n} l / 2\right)} h_{n}^{I}(y)
$$

where $\epsilon_{n}$ is the Neumann symbol, defined by

$$
\epsilon_{n}= \begin{cases}1, & n=0 \\ 2, & n \geq 1\end{cases}
$$

The $y$ variation of the eigenmodes in this region is given by

$$
h_{n}^{I}(y)=\frac{1}{b^{1 / 2}} \cos \lambda_{n} y
$$

where

$$
\lambda_{n}=\frac{n \pi}{b}
$$

and

$$
b=\frac{h}{2}+d .
$$

Finally the parameters $k_{n}$, which are either purely real or purely imaginary, are defined by

$$
k_{n}= \begin{cases}\left(\lambda_{n}^{2}-K^{2}\right)^{1 / 2}, & \text { if } \lambda_{n} \geq K \\ -\mathrm{i}\left(K^{2}-\lambda_{n}^{2}\right)^{1 / 2}=-\mathrm{i} k_{n}^{\prime}, & \text { if } \lambda_{n}<K .\end{cases}
$$

In the region $x \geq l / 2, \phi$ has the form

$$
\phi=-\sum_{n=0}^{\infty} \frac{\epsilon_{n} U_{n}^{I I} \mathrm{e}^{-j_{n}(x-l / 2)}}{j_{n}} h_{n}^{I I}(y)
$$

where

$$
j_{n}= \begin{cases}\left(\mu_{n}^{2}-K^{2}\right)^{1 / 2}, & \text { if } \mu_{n} \geq K \\ -\mathrm{i}\left(K^{2}-\mu_{n}^{2}\right)^{1 / 2}, & \text { if } \mu_{n}<K\end{cases}
$$

and

$$
\mu_{n}=\frac{2 n \pi}{h} .
$$

The $y$ variation of the eigenmodes in this region is given by

$$
h_{n}^{I I}(y)=\left(\frac{2}{h}\right)^{1 / 2} \cos \mu_{n} y .
$$

The sets $\left\{h_{n}^{I}(y)\right\}$ and $\left\{h_{n}^{I I}(y)\right\}$ are orthogonal on $[0, b]$ and $[0, h / 2]$ respectively.

The horizontal velocity on the interface between the regions at $x=l / 2$ is assumed to be continuous and is defined to be $U(y)$. Expressions for $U(y)$ in terms of the two sets of coefficients $\left\{U_{n}^{I}\right\}$ and $\left\{U_{n}^{I I}\right\}$ may be obtained by differentiation of each eigenfunction 
expansion with respect to $x$, and are given by

$$
U(y)=\sum_{n=0}^{\infty} \epsilon_{n} U_{n}^{I} \tanh \left(k_{n} l / 2\right) h_{n}^{I}(y)=\sum_{n=0}^{\infty} \epsilon_{n} U_{n}^{I I} h_{n}^{I I}(y) .
$$

Multiplication of (2.16) by each of the members of the sets of functions $\left\{h_{n}^{I}(y)\right\}$ and $\left\{h_{n}^{I I}(y)\right\}$ in turn, leads to the relations

$$
U_{m}^{I} \tanh \left(k_{m} l / 2\right)=\int_{0}^{h / 2} U(y) h_{m}^{I}(y) \mathrm{d} y
$$

and

$$
U_{m}^{I I}=\int_{0}^{h / 2} U(y) h_{m}^{I I}(y) \mathrm{d} y
$$

In order for a trapped mode to exist there must be no radiation of waves along the guide, so in (2.12), the coefficients of any of the modes which are oscillatory in $x$ must be zero. If the wave number is restricted to lie below the cut-off frequency for the propagation of the second symmetric mode along the guide, i.e. $K h<2 \pi$, then this is simply a requirement that

$$
U_{0}^{I I}=0
$$

As $h_{0}^{I I}(y)=h_{0}^{I}(y)(2.17),(2.18)$ and (2.19) immediately give

$$
U_{0}^{I} \tanh \left(k_{0} l / 2\right)=0,
$$

which means that either the coefficient of the first oscillatory term in the inner region must be zero or the wave number of the trapped mode, scaled by the cavity width, must be an even multiple of $\pi$, i.e.

$$
U_{0}^{I}=0 \quad \text { or } \quad K l=2 n \pi, \quad n \text { integer. }
$$

Application of continuity of pressure, i.e. $\phi$ on $x=l / 2,0<y<h / 2$ yields a second set of equations relating the coefficients in the two regions, namely

$$
\sum_{n=0}^{\infty} \epsilon_{n}\left[\frac{U_{n}^{I}}{k_{n}} h_{n}^{I}(y)+\frac{U_{n}^{I I}}{j_{n}} h_{n}^{I I}(y)\right]=0
$$

Under the assumption that there are a maximum of $N+1$ oscillatory terms in the inner region, this last set of equations can be written in terms of $U(y)$ with the use of $(2.17)$, (2.18) and (2.19) as

$$
\begin{gathered}
\sum_{n=N+1}^{\infty} \frac{2 \operatorname{coth}\left(k_{n} l / 2\right)}{k_{n}} h_{n}^{I}(y) \int_{0}^{h / 2} U\left(y^{\prime}\right) h_{n}^{I}\left(y^{\prime}\right) \mathrm{d} y^{\prime} \\
+\sum_{n=1}^{\infty} \frac{2}{j_{n}} h_{n}^{I I}(y) \int_{0}^{h / 2} U\left(y^{\prime}\right) h_{n}^{I I}\left(y^{\prime}\right) \mathrm{d} y^{\prime} \\
=-\sum_{n=0}^{N} \epsilon_{n} \frac{U_{n}^{I}}{k_{n}} h_{n}^{I}(y)
\end{gathered}
$$

The horizontal velocity may then be expanded as

$$
U(y)=-\sum_{n=0}^{N} \epsilon_{n} \frac{U_{n}^{I}}{k_{n}} v_{n}(y)
$$

and this leads to integral equations for each of the functions $v_{n}(y)$ of the form

$$
\int_{0}^{h / 2} K\left(y, y^{\prime}\right) v_{n}\left(y^{\prime}\right) \mathrm{d} y^{\prime}=h_{n}^{I}(y)
$$


where the kernel of each integral equation is identical and given by the positive definite function

$$
K\left(y, y^{\prime}\right)=2\left[\sum_{n=N+1}^{\infty} \frac{\operatorname{coth}\left(k_{n} l / 2\right)}{k_{n}} h_{n}^{I}(y) h_{n}^{I}\left(y^{\prime}\right)+\sum_{n=1}^{\infty} \frac{h_{n}^{I I}(y) h_{n}^{I I}\left(y^{\prime}\right)}{j_{n}}\right] .
$$

Multiplication of $(2.24)$ by $h_{m}^{I}(y), m=0, \ldots, N$, integration over the interval $0<y<$ $h / 2$ and comparison with (2.17) and (2.20) yields $N+1$ homogeneous equations for the $N+1$ coefficents $U_{n}^{I}=k_{n}^{\prime} W_{n}^{I}, n=0, \ldots, N$, which can be written as

$$
W_{m}^{I} k_{m}^{\prime} \sin \left(k_{m}^{\prime} l / 2\right)=\cos \left(k_{m}^{\prime} l / 2\right) \sum_{n=0}^{N} \epsilon_{n} W_{n}^{I} \int_{0}^{h / 2} v_{n}(y) h_{m}^{I}(y) \mathrm{d} y, \quad m=1, \ldots, N
$$

and

$$
0=\sum_{n=0}^{N} \epsilon_{n} W_{n}^{I} \int_{0}^{h / 2} v_{n}(y) \mathrm{d} y .
$$

Equations (2.21), (2.27) and (2.28) are side conditions which have to be satisfied by the coefficients $W_{n}^{I}, n=0, \ldots, N$ in order for a trapped mode to exist, once the functions $v_{n}(y)$ have been determined from (2.25) for a specific geometric configuration. If $K l=2 n \pi$ then (2.21) is satisfied automatically and (2.27) and (2.28) are satisfied if the resulting system of equations for $\left\{W_{n}^{I}\right\}$ has a zero of determinant. In general this only happens for specific sizes of indentations, so if the depth of indentation relative to the duct width, $d / h$, is given then zeros of determinant only occur for specific values of $l / h$. If $K l \neq 2 n \pi$ then $(2.21)$ gives $U_{0}^{I}=W_{0}^{I}=0$ and then (2.27) and (2.28) become $N+1$ homogeneous equations in $N$ unknowns, and these only have a non-zero solution if the rank of the system is less than or equal to $N-1$. For a fixed value of $d / h$ this only happens for discrete pairs of values of $K l$ and $l / h$. In both of these scenarios this is equivalent to saying that the indentation depth may be chosen freely $(d / h>1)$ but then trapped modes occur only for discrete pairings $(l / h, K h)$.

The integral equations in (2.25) are solved with the Galerkin method used by Evans \& Fernyhough (1995), whereby the functions $v_{n}(y)$ are approximated in terms of a series of functions which correctly model the singularity in the velocity at the corner of the indentation. Thus $v_{n}(y)$ is written as

$$
v_{n}(y)=\sum_{m=0}^{M} a_{m}^{n} w_{m}(y)
$$

where

$$
w_{m}(y)=\frac{(2 m) ! \Gamma(1 / 6) b^{1 / 2}(h / 2)^{-1 / 3}}{(-1)^{m} \pi \Gamma(2 m+1 / 3)\left((h / 2)^{2}-y^{2}\right)^{1 / 3}} C_{2 m}^{1 / 6}\left(\frac{2 y}{h}\right),
$$

$C_{n}^{\nu}(\cos \theta)$ are the ultra-spherical Gegenbauer polynomials and $\Gamma(z)$ is the gamma function. Substitution of this expansion into (2.25), multiplication by $w_{i}(y)$ and integration over the interval $0<y<h / 2$ yields the following system of equations for the $a_{m}^{n}$

$$
\sum_{m=0}^{M} K_{i m} a_{m}^{n}=F_{i n}, \quad 0 \leq i \leq M
$$


where

$$
\begin{aligned}
K_{i m}=\frac{2}{(2 \pi)^{1 / 3}} & {\left[\left(\frac{2 b}{h}\right)^{1 / 3} \sum_{n=N+1}^{\infty} \frac{\operatorname{coth}\left(k_{n} l / 2\right)}{n^{1 / 3} k_{n}} J_{2 i+1 / 6}(n \pi h /(2 b)) J_{2 m+1 / 6}(n \pi h /(2 b))\right.} \\
+ & \left.\frac{2 b}{h} \sum_{n=1}^{\infty} \frac{1}{n^{1 / 3} j_{n}} J_{2 i+1 / 6}(n \pi) J_{2 m+1 / 6}(n \pi)\right]
\end{aligned}
$$

$J_{\nu}(z)$ is a Bessel function of the first kind, and

$$
F_{i n}= \begin{cases}\frac{J_{2 i+1 / 6}(n \pi h /(2 b))}{(n \pi h / b)^{1 / 6}}, & n \neq 0, \\ \frac{1}{2^{1 / 3} \Gamma(7 / 6)}, & n=i=0, \\ 0 & n=0, i>0 .\end{cases}
$$

A NAG routine for numerical iteration is used to determine the size of the indentation for which embedded trapped modes exist. For each fixed value of $d / h$ an initial estimate of the geometric parameter $l / h$ and the scaled wavenumber $K l$ is made, based on an asymptotic approximation in which the depth of the indentation is assumed large compared to the duct width, i.e. $d / h \gg 1$. In this limit an embedded trapped mode may be represented by a function which is a localised oscillation in the rectangular cavity region and is zero outside in the duct region. In order for these two solutions to be matched smoothly over the interface between the regions (to a first approximation assumed to be a point) both the function and its horizontal derivative must be zero at that point. For the function to be non-trivial, the frequency must correspond to a double eigenvalue of an enclosed rectangular cavity of depth $b$ and width $l$.

The enclosed cavity modes which are symmetric in $x$ are given by

$$
\phi_{c}=\cos \frac{2 n \pi x}{l} \cos \frac{m \pi y}{b}, m, n, \text { integer, }
$$

and so double eigenvalues are only possible for a discrete set of values of $l / b$ given by

$$
\frac{l}{b}=2\left[\frac{n^{2}-p^{2}}{q^{2}-m^{2}}\right]^{1 / 2}, \quad m, n, p, q \text { integer, } p<n, m<q .
$$

The corresponding eigenvalues are given by

$$
K l=\pi\left[4 n^{2}+\frac{m^{2} l^{2}}{b^{2}}\right]^{1 / 2}
$$

and the values in (2.35) and (2.36) act as initial estimates for the full numerical procedure. It is worth noting that if $m=0,(2.36)$ gives $K l=2 n \pi$ which corresponds to an exact solution of the side condition (2.21). Results for the rectangular indentation will be presented in section 6 .

\section{Resonances of thin objects in a duct: Parker mode problem}

The classical problem of a laterally bounded thin object is an infinitely thin plate of finite length located parallel to the duct walls on the centreline of the duct, cf. figure 1a. Parker (1966) was the first to experimentally observe acoustic resonances in such a system and computed the trapped modes, now termed Parker modes, via a numerical 
relaxation technique, Parker (1967). The leaky Parker modes above the first cut-off frequency were computed first independently by Duan (2004) and Hein et al. (2004). Duan (2004) used the mode matching method and demonstrated the existence of leaky mode resonances with zero radiation loss for the Neumann problem. Hein et al. (2004) formulated an eigenvalue problem using a numerical method with PML boundary conditions but did not pay any attention to the possible existence of embedded trapped modes. Both the Neumann and the Dirichlet problem were treated. Individual resonant modes can be identified by two integers $(m, n)$, where $m$ denotes the number of nodal lines in the direction normal to the duct axis and $n$ is the number of nodal lines parallel to the axis, cf. Koch (1983). Parker (1967) named the first four trapped modes $\alpha, \beta, \gamma$ and $\delta$ mode.

McIver et al. (2001), McIver et al. (2002), Linton et al. (2002) and Duan (2004) were the first to compute isolated trapped modes above the first cut-off frequency for a finite-length plate of length $l$ placed on the centreline of a Neumann duct. These isolated trapped modes are called embedded trapped modes because their eigenvalues are embedded in the continuous spectrum. The corresponding embedded trapped modes for the Dirichlet problem were published by Linton \& Ratcliffe (2004). All these embedded trapped modes exist only for specific plate lengths and frequencies in the frequency range $1<\operatorname{Re}(K / 2 \pi)<2$ and are compared with the leaky mode resonances in figures 2 and 3. All computed solutions are antisymmetric about the duct axis.

In addition to the real part of the resonant frequencies, presented in Hein et al. (2004), the imaginary part of a few leaky Neumann and Dirichlet resonances is plotted in figure 3 with the corresponding modal numbers marked on the right-hand side of figure 3 . Furthermore, the computation of leaky mode resonances is extended up to $\operatorname{Re}(K / 2 \pi)=$ 3. The solid lines depict resonances which are symmetric in $x$, dashed lines represent resonances which are antisymmetric in $x$. We see that the embedded trapped modes lie exactly on the resonance curves of the leaky modes for $1<\operatorname{Re}(K / 2 \pi)<2$ where the imaginary part $\operatorname{Im}(K / 2 \pi)$ vanishes. Linton et al. (2002) computed the symmetric embedded trapped modes for the Neumann problem while Duan (2004) presented both the symmetric and antisymmetric embedded trapped modes. Duan (2004) also computed the complex leaky mode resonances for the Neumann problem and plotted them together with the embedded trapped modes. Figures 2 and 3 demonstrate clearly that embedded trapped mode frequencies can be found by numerically computing the complex resonances and searching for those resonant frequencies where the imaginary part of the leaky modes vanishes (within numerical accuracy). Above $\operatorname{Re}(K / 2 \pi)=2$ apparently no embedded modes are possible. This suggests that for each cut-on mode an additional geometric parameter is needed to cancel it in order to make the radiation loss zero. With $l / h$ only one such parameter is available and therefore embedded trapped modes are posssible only in $1<\operatorname{Re}(K / 2 \pi)<2$. But figure 3 shows that for $\operatorname{Re}(K / 2 \pi)>2$ almost trapped modes occur at particular $l / h$ with rather small imaginary parts $\operatorname{Im}(K / 2 \pi)$. Therefore, aside from the embedded trapped modes such almost trapped modes are also of physical importance because the system can be excited more easily at these frequencies.

Figure 4 shows eigenfunctions $\operatorname{Re}(\phi)$ for selected trapped modes in figure 2 . The thin lines outline the individual rectangular domains up to the PML. The actual computations were made by using only a quarter of the domain and taking into account the appropriate symmetries, i.e. NSA, NAA, DSA and DAA. Starting with Parker's trapped $\alpha$ and $\gamma$ mode in figure $4 \mathrm{a}$ and $4 \mathrm{~b}$ the selected embedded trapped modes in figure $4 \mathrm{c}-4 \mathrm{~h}$ display more and more complicated patterns but follow a systematic rule. 


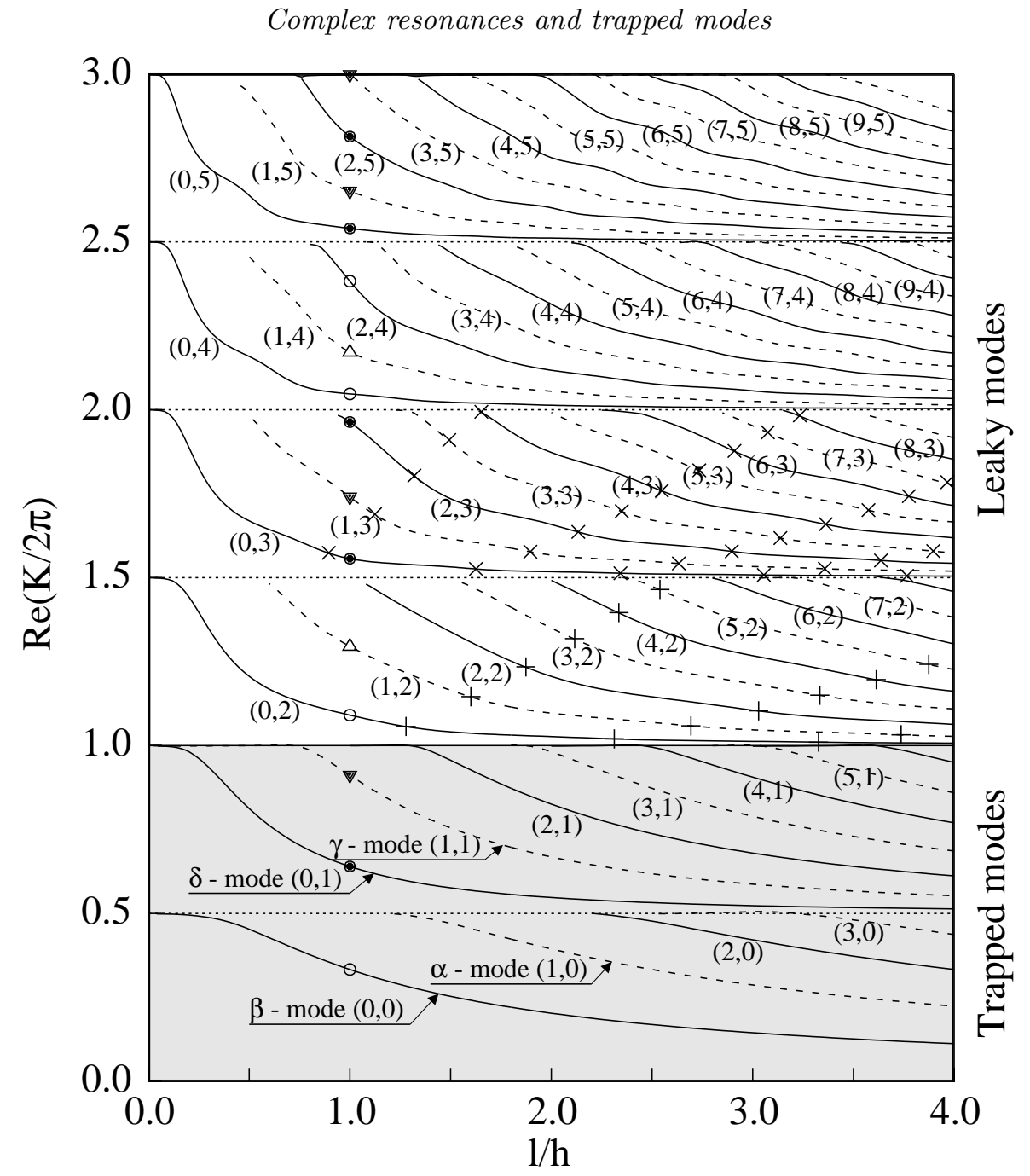

Figure 2. Parker mode resonances with trapped and embedded trapped modes: real part of resonant frequencies $\operatorname{Re}(K / 2 \pi)$ as function of $l / h$. The shaded area marks Parker's trapped mode domain. + embedded trapped Neumann modes of Duan (2004), $\times$ embedded trapped Dirichlet modes of Linton \& Ratcliffe (2004).

\section{Resonances of thick objects in a duct}

A typical model for a thick object in a duct is a single circular or elliptical cylinder centred on the channel axis, cf. figure 1b. For such a configuration the existence of trapped modes below the first cut-off frequency was established by Callan et al. (1991). Linton \& Evans (1992) found trapped modes for cylinders of fairly general cross-section centred on the channel axis and Evans et al. (1994) proved that there exists at least one trapped mode anti-symmetric about the duct centreline. Following the discovery of Maniar \& Newman (1997) that wave diffraction forces reach large amplitudes for certain frequencies above the first cut-off frequency Evans \& Porter (1998) showed convincingly that an isolated trapped mode exists above the first cut-off frequency for a circular cylinder placed on the centreline of the duct. McIver et al. (2001) demonstrated that the isolated embedded trapped mode of Evans \& Porter (1998) is part of a whole branch of embedded 

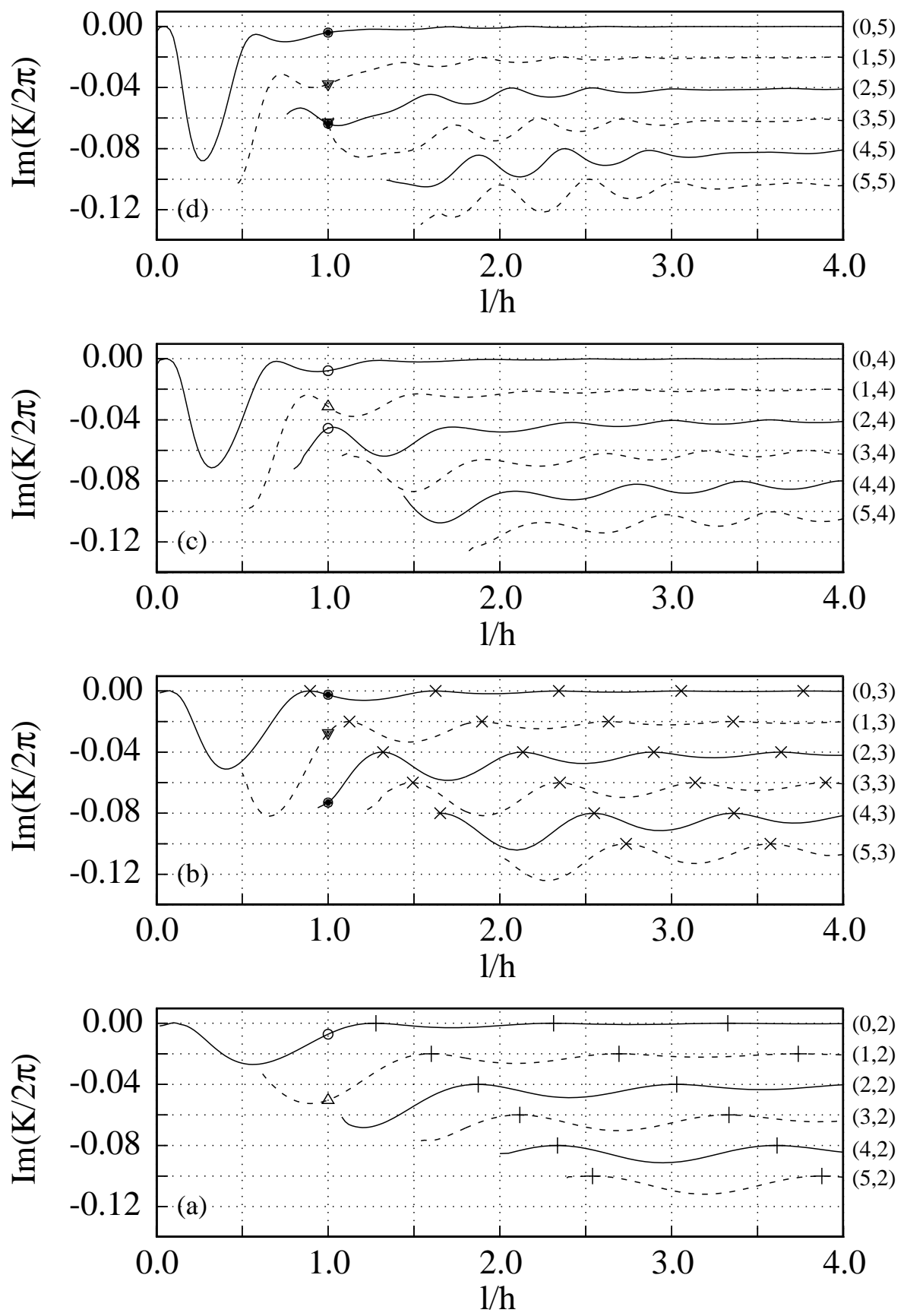

Figure 3. Parker mode resonances with embedded trapped modes: imaginary part of resonant frequencies $\operatorname{Im}(K / 2 \pi)$ as function of $l / h$. (a) Neumann problem for $1<\operatorname{Re}(K / 2 \pi)<1.5$. (b) Dirichlet problem for $1.5<\operatorname{Re}(K / 2 \pi)<2$. (c) Neumann problem for $2<\operatorname{Re}(K / 2 \pi)<2.5$. (d) Dirichlet problem for $2.5<\operatorname{Re}(K / 2 \pi)<3$. (To distinguish the individual curves the origin of the ordinate of each curve is shifted by -0.02$)$. + embedded trapped Neumann modes of Duan (2004), × embedded trapped Dirichlet modes of Linton \& Ratcliffe (2004). 
$(\mathrm{g})$

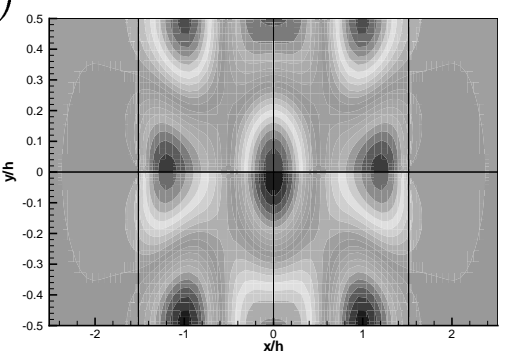

(e)

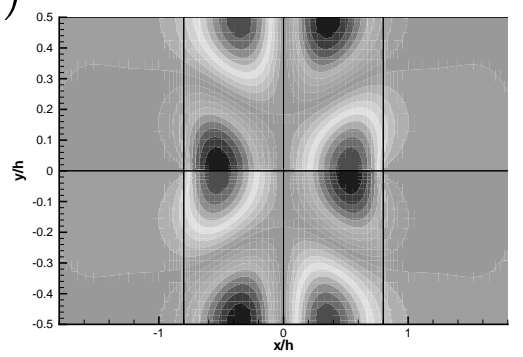

(c)

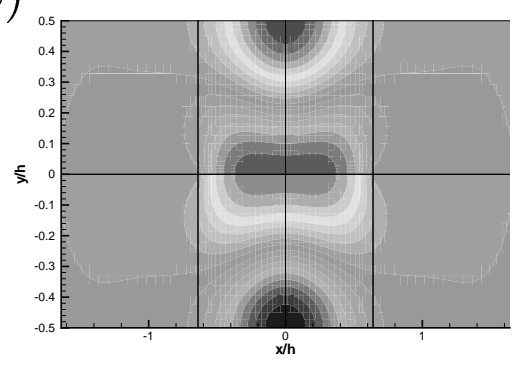

(a)

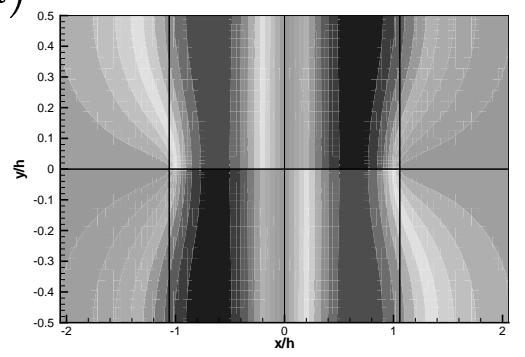

(h)

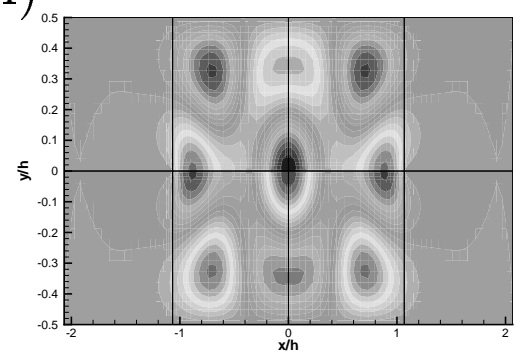

(f)

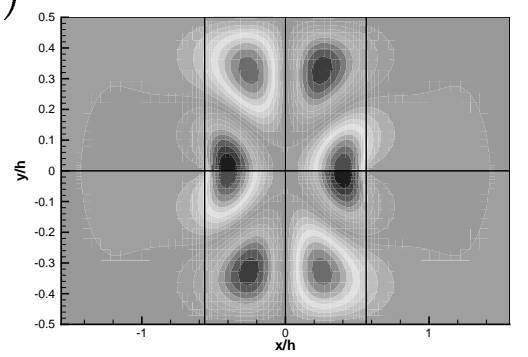

(d)

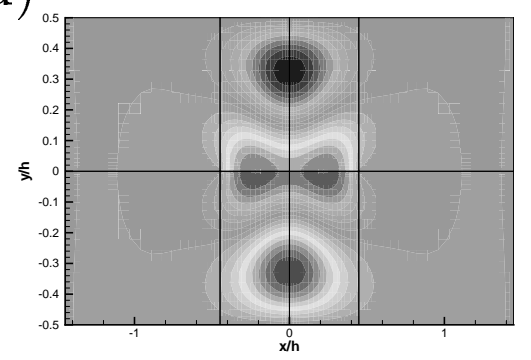

(b)

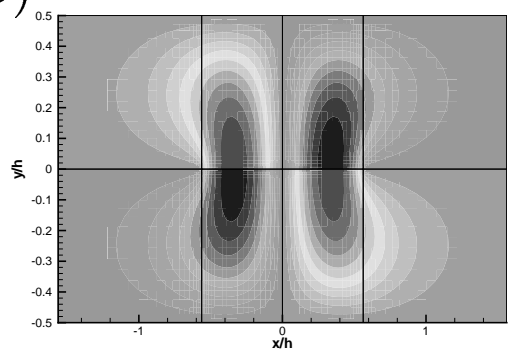

Figure 4. Selected eigenfunctions $\operatorname{Re}(\phi)$ of trapped modes about a flat plate centred in a duct: (a) Parker's $\alpha$ mode NAA at $l / h=1.874, K / 2 \pi=0.379$, (b) Parker's $\gamma$ mode DAA at $l / h=1.896, K / 2 \pi=0.683$, and embedded trapped modes: (c) NSA at $l / h=1.279, K / 2 \pi=1.057$, (d) DSA at $l / h=0.896, K / 2 \pi=1.574$, (e) NAA at $l / h=1.601, K / 2 \pi=1.145$, (f) DAA at $l / h=1.125, K / 2 \pi=1.689$, (g) NSA at $l / h=3.029, K / 2 \pi=1.103,(\mathrm{~h}) \mathrm{DSA}$ at $l / h=2.134, K / 2 \pi=1.636$. 


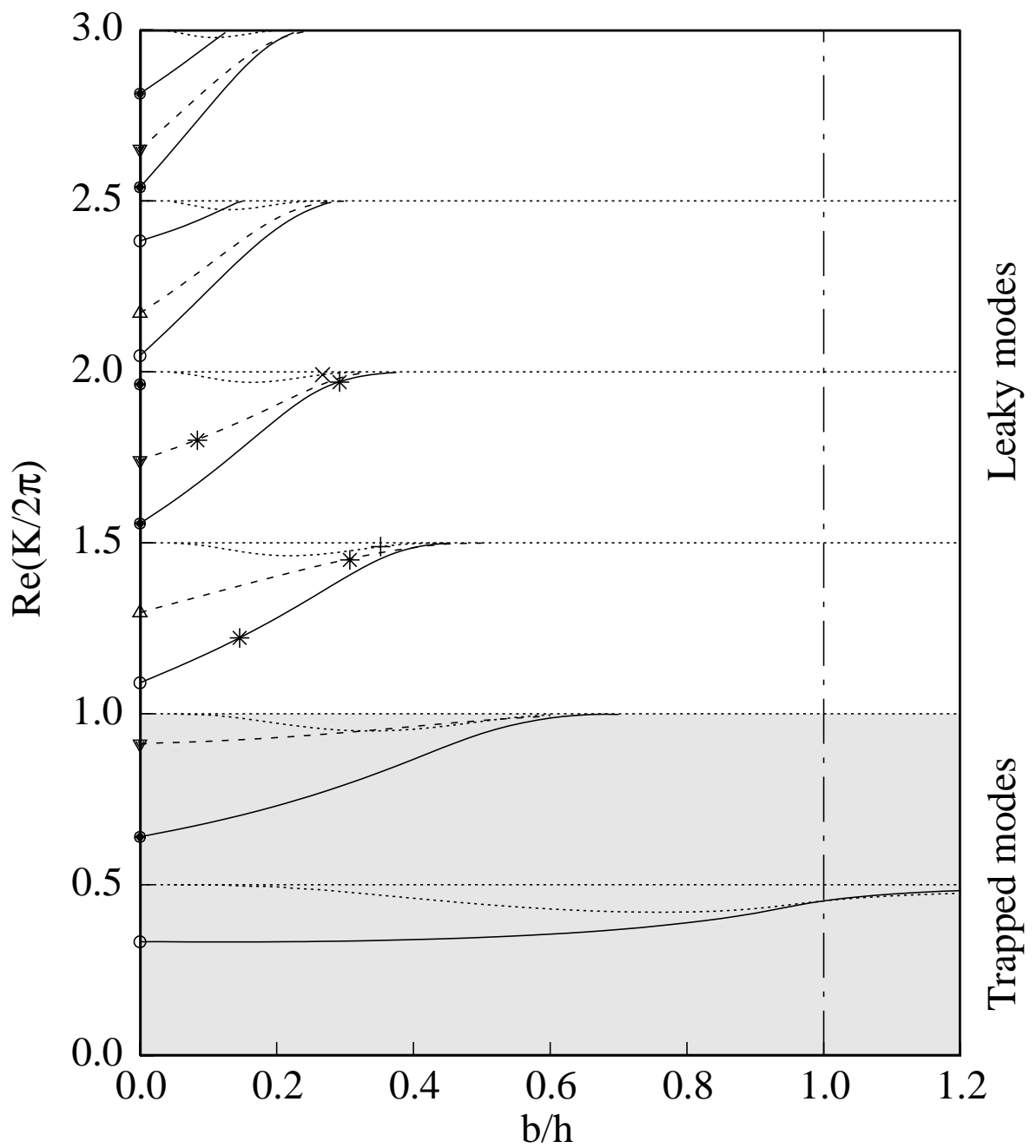

FIGURE 5. Resonances of single circular and elliptical cylinder $(a / h=1)$ centred in a duct of height $h$ with trapped and embedded trapped modes: real part of resonant frequencies $\operatorname{Re}(K / 2 \pi)$ as function of $b / h$. The shaded area marks the trapped mode domain. Dotted curves: $x$-symmetric resonances of circular cylinder with $b=a$. Full lines and dashed lines: $x$-symmetric and $x$-antisymmetric resonances of elliptical cylinder. + and $\times$ : Neumann and Dirichlet isolated trapped mode of Evans \& Porter (1998) for circular cylinder. * embedded trapped Neumann and Dirichlet modes for elliptical cylinder including the NSA embedded trapped mode found by Linton et al. (2002).

trapped modes if an additional geometrical parameter is introduced. In the follow-up paper Linton et al. (2002) extended the computation of trapped and embedded trapped modes to obstacles which are no longer symmetric about the duct centreline.

Figures 5 and 6 show the complex resonances for circular and elliptical cylinders. Only resonances antisymmetric about the duct axis are considered. For elliptical cylinders $a / h=1$ is kept constant and $b / h$ is varied, with $a$ being the major axis of the elliptical cylinder in channel direction and $b$ the minor axis normal to the channel. The elliptical cylinder is aligned with the duct centreline. For the circular cylinder $b \equiv a$ is the diameter and $b / h$ is varied. Embedded trapped modes have zero radiation loss and 

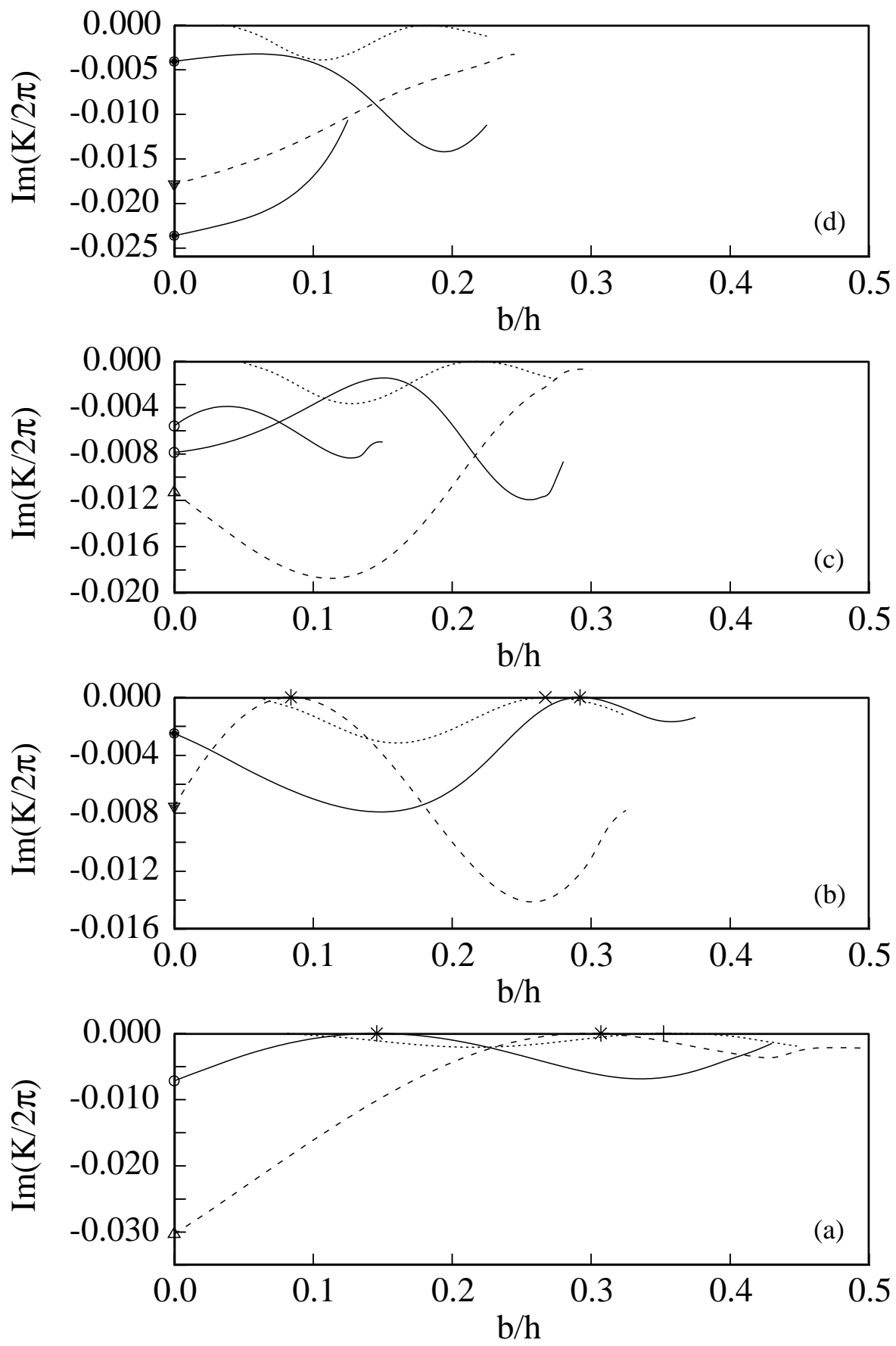

Figure 6 . Resonances of single circular and elliptical cylinder $(a / h=1)$ centred in a duct of height $h$ with embedded trapped modes: imaginary part of resonant frequencies $\operatorname{Im}(K / 2 \pi)$ as function of $b / h$. (a) Neumann problem for $1<\operatorname{Re}(K / 2 \pi)<1.5$. (b) Dirichlet problem for $1.5<\operatorname{Re}(K / 2 \pi)<2$. (c) Neumann problem for $2<\operatorname{Re}(K / 2 \pi)<2.5$. (d) Dirichlet problem for $2.5<\operatorname{Re}(K / 2 \pi)<3$. Dotted curves: $x$-symmetric resonances of circular cylinder with $b=a$. Full lines and dashed lines: $x$-symmetric and $x$-antisymmetric resonances of elliptical cylinder. + and $\times$ : Neumann and Dirichlet isolated trapped mode of Evans \& Porter (1998) for circular cylinder. * embedded trapped Neumann and Dirichlet modes for elliptical cylinder including the NSA embedded trapped mode found by Linton et al. (2002). 
therefore correspond to those resonances whose imaginary part is equal to zero. In our numerical computation we can establish this only within numerical accuracy. This way we recover the Neumann and Dirichlet isolated trapped mode of Evans \& Porter (1998) for the circular cylinder marked by the plus and times symbol respectively in figures 5 and 6 .

For $2<\operatorname{Re}(K / 2 \pi)<3$ resonances with very small $\operatorname{Im}(K / 2 \pi)$ are also observed. However, these are probably near-trapped modes and not genuine trapped modes, and the corresponding approximate values of $\operatorname{Re}(K / 2 \pi)$ are obtained from the genuine embedded modes as follows. From the surface elevation of the embedded Dirichlet eigenfunction in figure 4 in Evans \& Porter (1998), it is clear that there is a nearly horizontal streamline at $y \approx 0.75$. If the region between this line and the upper Dirichlet boundary of the guide is reflected in the latter boundary, the resultant surface plot represents a trapped mode for a circular cylinder of the same radius $2 a / h=0.267$, at the same frequency, $K_{D} / 2 \pi=1.992$, in a Neumann guide with a nearly horizontal upper wall at $y=1.25$. If this boundary were completely flat and the guide were resized so that it lay at $y=1$, this mode would represent an embedded trapped mode for a cylinder of radius $2 a / h=0.267 / 1.25=0.21$ in a Neumann guide, with a mode frequency $K / 2 \pi=1.25 K_{D} / 2 \pi=2.49$. Further reflections will produce higher frequency near-trapped modes of alternately Dirichlet and Neumann type. At $b / h=1$ the circular and elliptical cylinder touch the duct walls, such that for $b / h>1$ the cylinders separate the duct into two independent semi-infinite domains. The value $b / h=1$ where for $a / h=1$ the elliptical cylinder becomes identical with the circular cylinder is marked by a dash-dotted line in figure 5 .

Similar to the finite-length plates in the Parker mode problem of section 3 more than one embedded trapped mode is possible for elliptical cylinders between two cut-off frequencies if $a / h$ is large enough. This becomes immediately clear if we let $b / h \rightarrow 0$ keeping $a / h$ fixed. In this limit the elliptical cylinder becomes an infinitely thin plate of length $a / h$. From figure 2 we see that depending on the value of $a / h$ several resonances can exist in a prescribed $\operatorname{Re}(K / 2 \pi)$ interval. For $a / h=1$ these resonances are marked by symbols in figures 2 and 3 . The same symbols are used in figures 5 and 6 at $b / h=0$, providing the starting values of the resonance curves for the elliptical cylinder. Open symbols correspond to Neumann resonances while filled symbols mark Dirichlet resonances. Circles denote modes symmetric in $x$ and triangles denote antisymmetric modes. As can be seen the agreement is very good providing a valuable test of our different codes, because the Parker mode resonances were computed with the multi-domain Chebyshev collocation code employed in Hein et al. (2004) while the resonances of this section were computed with the finite element code NGSolve of Joachim Schöberl as applied in Hein et al. (2005).

Varying $b / h$ for fixed $a / h$ the imaginary part of the frequency can reach zero for certain values of $b / h$ and $\operatorname{Re}(K / 2 \pi)>1$, i.e. the embedded trapped modes. For $a / h=1$ and for $1<\operatorname{Re}(K / 2 \pi)<1.5$ only one symmetric embedded trapped mode is possible which agrees exactly with the Neumann embedded trapped mode NSA at $b / h=0.145729, K / 2 \pi=1.221895$ found by Linton et al. (2002). Employing the method of Linton et al. (2002) the antisymmetric embedded trapped Neumann mode NAA at $b / h=0.307, K / 2 \pi=1.45$ and the symmetric and antisymmetric Dirichlet embedded trapped modes DSA at $b / h=0.292, K / 2 \pi=1.97$ and DAA at $b / h=0.084, K / 2 \pi=1.80$ respectively, were computed and are depicted by the asterisks in figures 5 and $6 \mathrm{a}$ and $6 \mathrm{~b}$. In all these cases the directly computed embedded trapped modes agree very well with the numerically computed resonances having a vanishingly small imaginary part.

Sample eigenfunctions of a symmetric and antisymmetric Dirichlet trapped mode around the elliptical cylinder are shown in figures $7 \mathrm{a}$ and $7 \mathrm{~b}$, whereas figures $7 \mathrm{c}-7 \mathrm{f}$ depict the eigenfunctions of the four embedded trapped modes. Only a quarter of the 
(e)

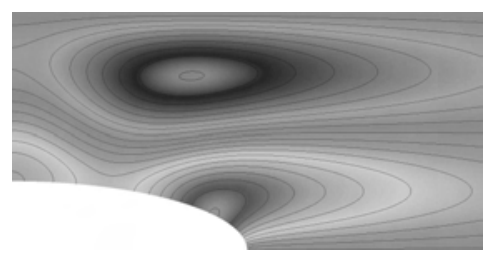

(c)

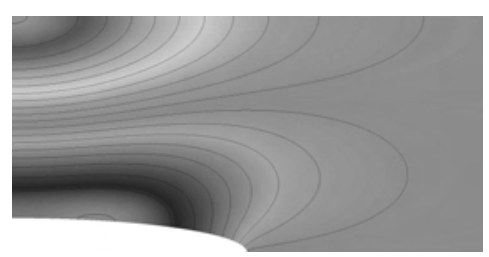

(a)

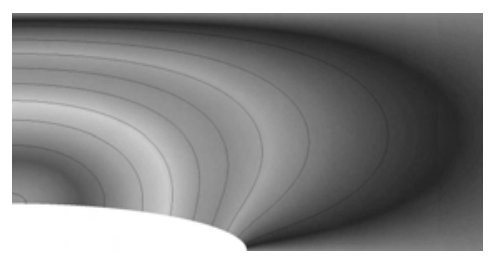

(f)

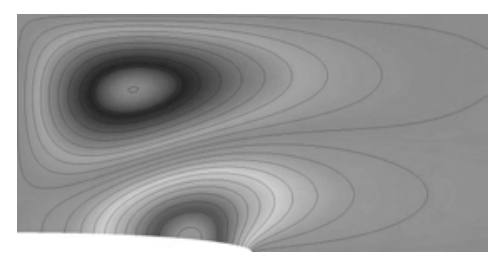

(d)

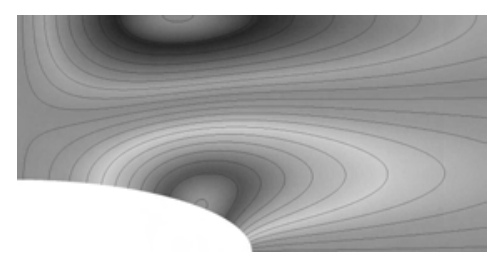

(b)

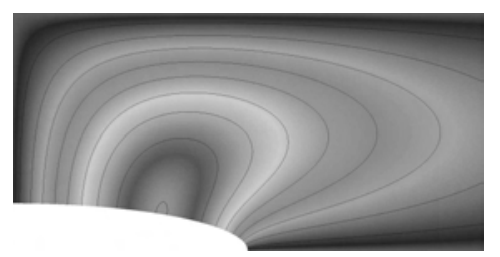

FIgURE 7. Eigenfunctions $R e(\phi)$ of two selected Dirichlet trapped modes of the elliptical cylinder in a duct with $a / h=1$ : (a) DSA at $b / h=0.2, K / 2 \pi=0.731$, (b) DAA at $b / h=0.2, K / 2 \pi=0.930$, and the four embedded trapped modes: (c) NSA at $b / h=0.146, K / 2 \pi=1.222$, (d) NAA at $b / h=0.307, K / 2 \pi=1.453$, (e) DSA at $b / h=0.292, K / 2 \pi=1.971$, (f) DAA at $b / h=0.084, K / 2 \pi=1.802$.

eigenfunctions is shown corresponding to the computational domain which takes advantage of the various symmetries. Near the cut-off frequencies it is difficult to distinguish the resonances from the discrete approximations of the continuous spectra in our numerical analysis, cf. the discussion of this problem in the next section. For frequencies above $\operatorname{Re}(K / 2 \pi)=2$ the maxima of $\operatorname{Im}(K / 2 \pi)$ for the resonances of the elliptical cylinder are clearly below zero and therefore belong to near-trapped modes.

\section{Resonances in a butterfly valve model}

Aslanyan et al. (2000) proved that for symmetric objects off centre in a duct the real trapped mode frequencies tranform into complex resonances. Examples are the off-centre plate of Evans et al. (1993) or the off-centre structures of Linton et al. (2002). The same is true for an ellipse under angle of attack $\alpha \neq 0^{\circ}$ which can be considered a simple model of a butterfly valve, cf. figure $1 \mathrm{~d}$. In figure 8 we plotted the real and imaginary part of the fundamental complex resonance for an ellipse with $a / h=1$ and $b / h=0.4$ and $\alpha$ varying between $\alpha=0^{\circ}$ and $\alpha=90^{\circ}$. Starting with the trapped mode of figure 5 for $\alpha=0^{\circ}$ the trapped mode turns into a complex resonance and reaches maximal damping between $\alpha=40^{\circ}$ and $50^{\circ}$. From this one concludes that unstable shear layers separating from the ellipse can excite a resonance in a butterfly valve most likely near the open or closed position and one expects the largest noise near those positions.

Figure 8 shows that near a cut-off frequency the resonance obviously depends on the 

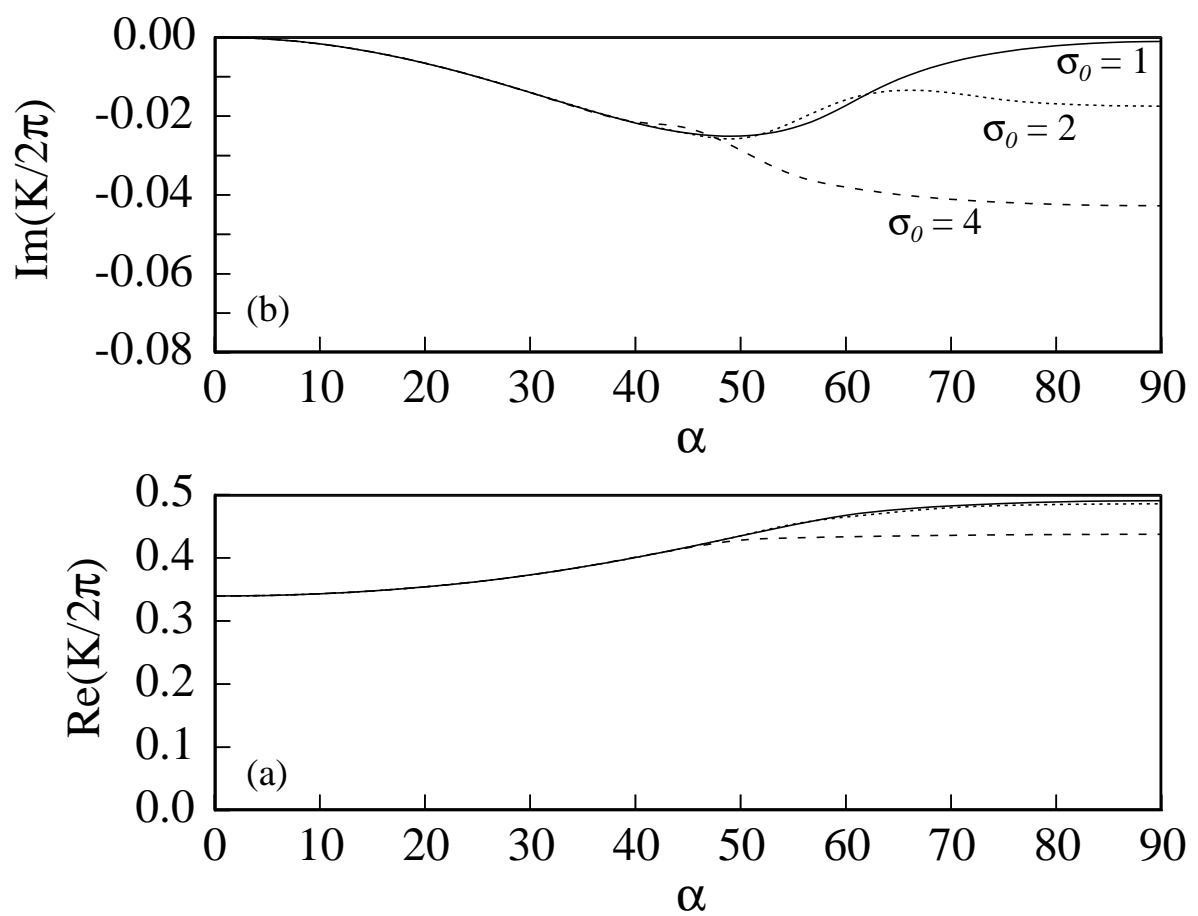

FiguRE 8. Resonances of elliptical cylinder in a duct $(a / h=1, b / h=0.4)$ as function of valve angle $\alpha$ : (a) real part and (b) imaginary part of fundamental complex resonance with $\sigma_{0}=1,2,4$.

PML parameter $\sigma_{0}$, a definite flaw of the complex scaling method. The reason behind this failure is demonstrated in figure 9 where the complex spectra are plotted as function of $\alpha$ for $\sigma_{0}=1,2$ and 4 . While the resonances (shown by the solid curve) should be independent of the chosen value of $\sigma_{0}$, and this is true up to $\alpha \approx 40^{\circ}$ in figure 9 , the continuous spectra depend strongly on $\sigma_{0}$. In our numerical approach these continuous spectra are approximated by discrete eigenvalues which can interact with a discrete resonance. For smaller values of $\sigma_{0}$ the continuous spectra remain near the cut-off frequency and an interaction with the resonance occurs only very close to the cut-off frequency, cf. figure 9a,b. For larger values of $\sigma_{0}$ the interaction occurs much earlier, cf. figure 9c, and can lead to completely wrong results as shown in figure 8 . From this we conclude that, contrary to the continuous spectrum near zero where a larger value of $\sigma_{0}$ resulted in more accurate resonances, cf. Hein et al. (2004), the continuous spectra near a duct cut-off frequency appear to be more accurate for smaller values of $\sigma_{0}$.

\section{Resonances of a rectangular duct indentation}

Evans \& Linton (1991) showed that trapped modes exist also near duct indentations below the first antisymmetric duct cut-off frequency. According to our nomenclature introduced in section 2.2 we distinguish between NSA or NAA modes, which are antisymmetric about the duct axis, and NSS or NAS modes, which are symmetric about the duct axis. All obey Neumann boundary conditions on the duct and cavity walls. NSA and NSS modes are symmetric in $x$ and NAA and NAS modes are antisymmetric in $x$. Non-embedded trapped modes are possible only for the antisymmetric NSA and NAA modes and can be excited by flow in ducts and pipes with side branches which are located 
Complex resonances and trapped modes
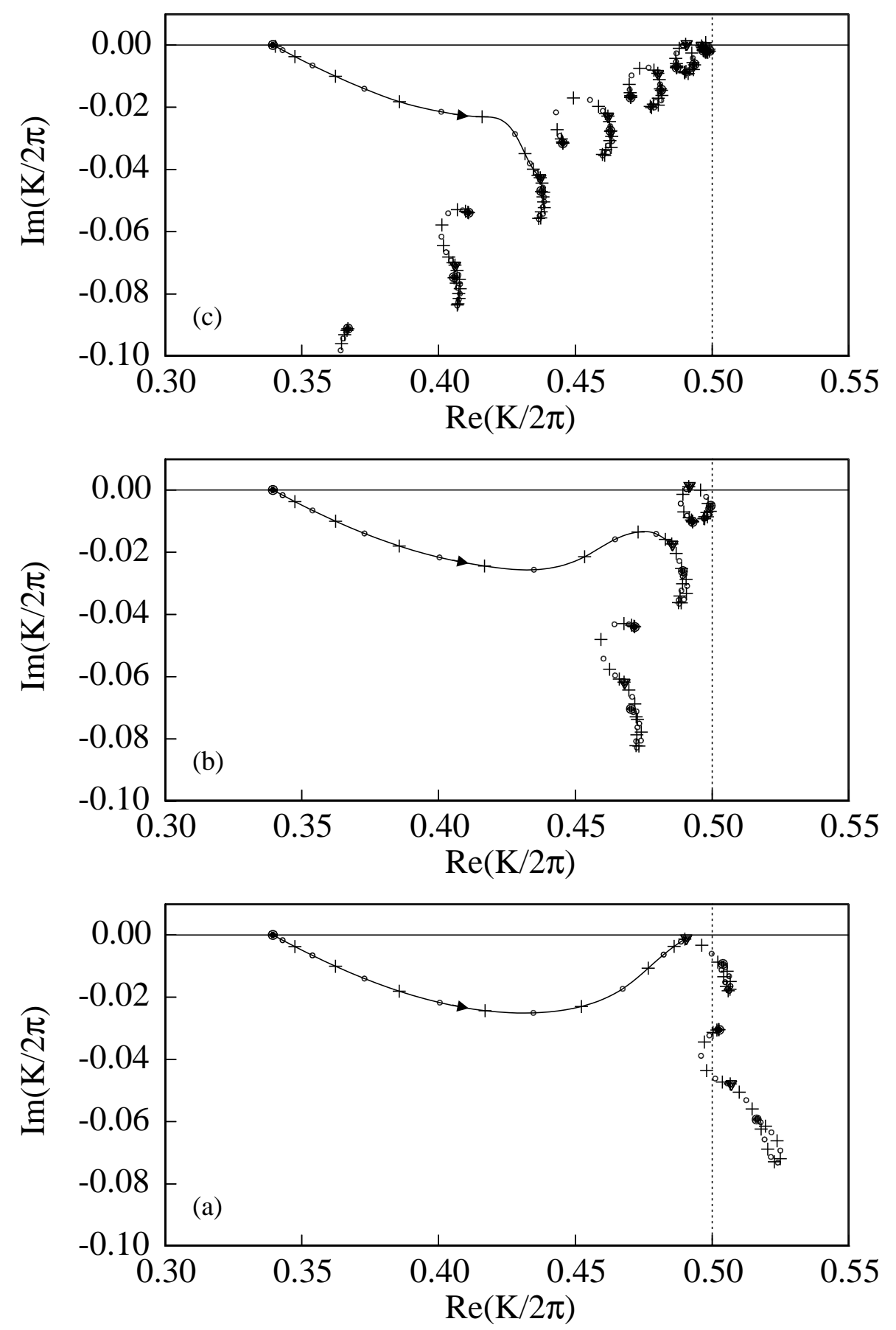

Figure 9. Complex resonances (solid curves with symbols) and continuous spectra (symbols only) of elliptical cylinder in a duct $(a / h=1, b / h=0.4)$ as function of valve angle $\alpha\left(\bullet \alpha=0^{\circ}\right.$; $\boldsymbol{\nabla} \alpha=90^{\circ}$ ) near first duct cut-off frequency: (a) $\sigma_{0}=1$, (b) $\sigma_{0}=2$, (c) $\sigma_{0}=4$. 


\begin{tabular}{ccccccccc}
\multicolumn{4}{c}{$N S S$} & \multicolumn{4}{c}{$N S A$} & \multicolumn{2}{c}{$N A S$} & \multicolumn{2}{c}{$N A A$} \\
$l / h$ & $K h / 2 \pi$ & $l / h$ & $K h / 2 \pi$ & $l / h$ & $K h / 2 \pi$ & $l / h$ & $K h / 2 \pi$ \\
& & & & & & & \\
1.476 & 0.678 & 0.846 & 1.197 & 2.074 & 0.723 & 1.309 & 1.167 \\
1.732 & 0.667 & 0.949 & 1.167 & 2.358 & 0.711 & 1.422 & 1.167 \\
2.552 & 0.784 & 1.214 & 0.842 & 2.958 & 0.845 & 1.759 & 0.868 \\
2.911 & 0.758 & 1.225 & 1.167 & 3.381 & 0.807 & 1.768 & 1.423 \\
2.978 & 0.672 & 1.463 & 1.379 & 3.628 & 0.689 & 1.894 & 1.167 \\
2.987 & 0.335 & 1.500 & 0.833 & 3.649 & 0.959 & 1.946 & 1.379
\end{tabular}

TABLE 1. Embedded modes for a rectangular indentation of depth $d / h=1$

symmetrically on both sides of the duct centre line, cf. Kriesels et al. (1995); Ziada \& Shine (1999); Dequand et al. (2003). Recently Sugimoto \& Imahori (2006) computed the frequency of trapped modes in a waveguide with Helmholtz resonantors analytically as root of an algebraic frequency equation. In his PhD thesis Duan (2004) showed for the first time that in addition to these trapped modes also isolated embedded trapped modes exist for rectangular indentations above the first cut-off frequency, cf. also Duan \& McIver (2002). These embedded trapped modes exist only for particular geometric parameters and at particular frequencies. The numerical resonance computations of Koch (2005) for rectangular indentations showed also resonances with vanishingly small damping, but the chosen parameters were quite different from those of Duan \& McIver (2002) and no direct comparison of the two methods was possible. One of the main objectives of the present work is to apply the two methods to one and the same problem in order to test whether the embedded trapped modes of Duan \& McIver (2002) correspond exactly to the resonances of Koch (2005) with vanishingly small imaginary part. Therefore, we chose the rectangular indentation of length $l$ and depth $d$ depicted in figure $1 \mathrm{c}$ and performed the comparison for $d / h=1$ as in the example of Koch (2005).

The results of this comparison for the antisymmetric NSA and NAA modes are shown in figure 10. The symbols mark the directly computed embedded modes (circles for NSA modes symmetric in $x$ and triangles for NAA modes antisymmetric in $x$ ) and the solid and dashed lines show the symmetric and antisymmetric resonances, respectively. One can see that the embedded trapped modes indeed correspond exactly to the resonances with vanishing imaginary part. For numerical comparison table 1 contains the values of the first six embedded modes ordered in terms of indentation width, for cavities of depth $d / h=1$, calculated using the method described in section 2.2.

The arrows on the right-hand side of figure 10a mark the cut-on frequencies $(2 n-$ $1) /[2(h+2 d)], n=1,2, \ldots$ of the first three NSA and NAA modes in the wider duct $2 b=h+2 d$ formed by the duct plus the two cavities on both sides of the duct. The damping $\operatorname{Im}(K / 2 \pi)$ of some selected antisymmetric and symmetric modes, as denoted on the right-hand side of the figures, is shown in figures $10 \mathrm{~b}$ and $10 \mathrm{c}$, respectively. For better distinction the $y$ axis of each damping curve is shifted by -0.02 . All modes have zero damping as long as they are in the shaded trapped mode domain below the cut-off frequency of the first antisymmetric mode in the duct. As suggested by East (1966), for $l / h \rightarrow 0$ the resonances approach the one-dimensional organ pipe resonances $(2 n-1) /(4 d), n=1,2, \ldots$ marked by arrows on the left-hand side of figure 10a. Figure 11 shows eigenfunctions $\operatorname{Re}(\phi)$ for selected antisymmetric trapped and embedded trapped cavity modes.

Duan (2004) also identified embedded NSS and NAS trapped modes which are sym- 

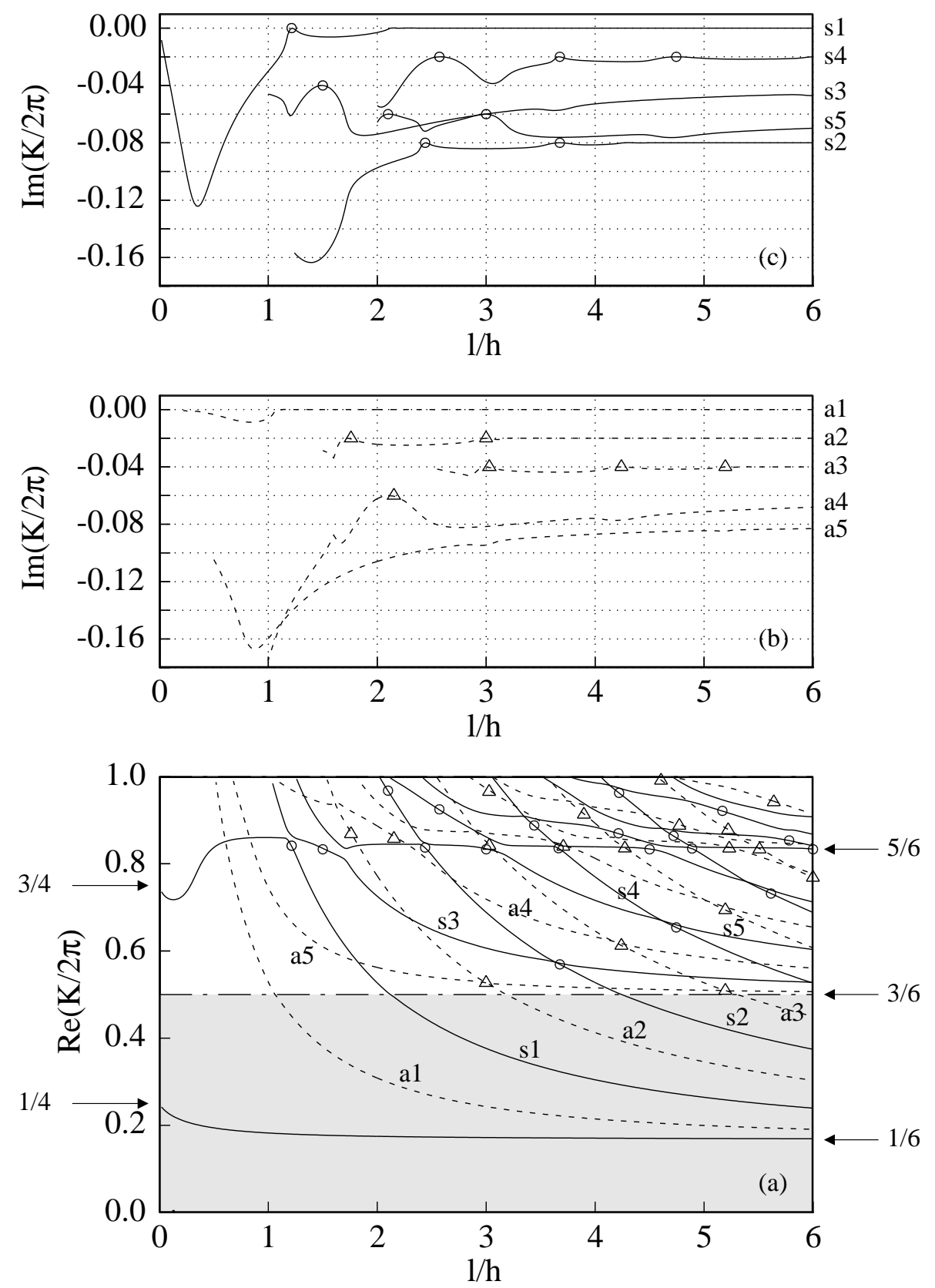

FIgURE 10. NSA and NAA resonances and embedded trapped modes for rectangular cavity in duct with $d / h=1$ : (a) Variation of resonant frequency $R e(K / 2 \pi)$ with $l / h$. (b) Variation of resonance damping $\operatorname{Im}(K / 2 \pi)$ of NAA modes with $l / h$. (c) Variation of resonance damping $\operatorname{Im}(K / 2 \pi)$ of NSA modes with $l / h$. (To distinguish the individual damping curves the origin of the ordinate of each curve is shifted by -0.02). Shaded area marks domain of trapped modes, $\circ$ embedded trapped NSA modes, $\triangle$ embedded trapped NAA modes. 
(e)

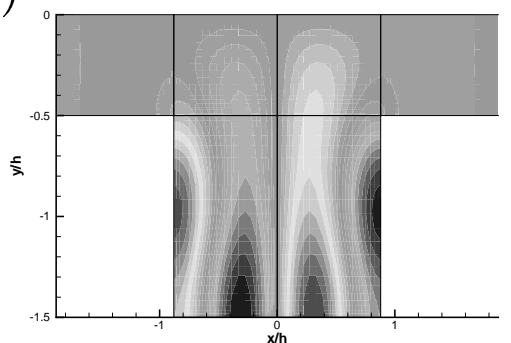

(c)

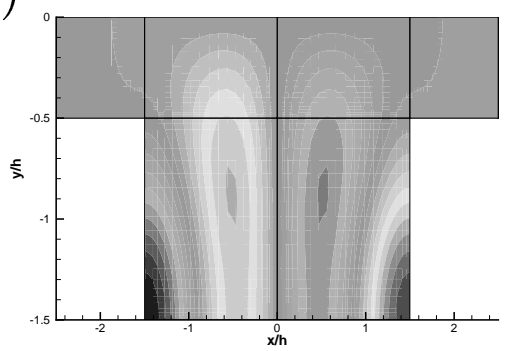

(a)

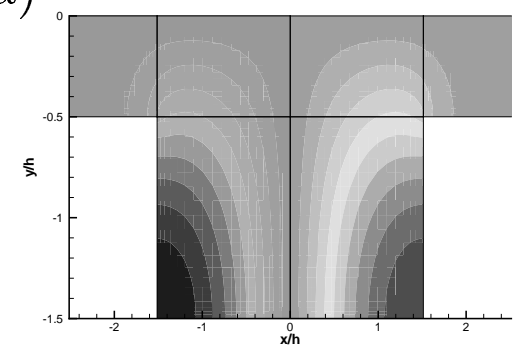

(f)

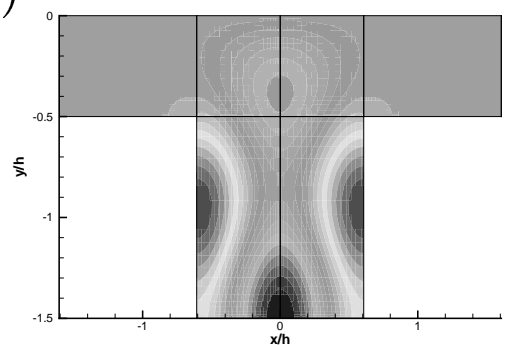

(d)

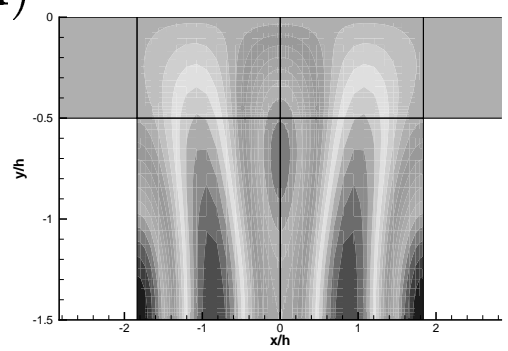

(b)

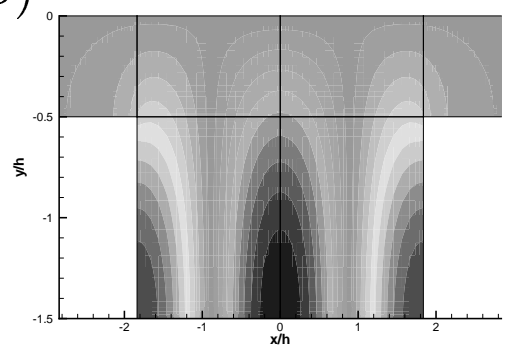

Figure 11. Selected eigenfunctions $R e(\phi)$ of antisymmetric trapped modes: (a) NAA at $l / h=3.028, K / 2 \pi=0.242$, (b) NSA at $l / h=3.676, K / 2 \pi=0.323$, and embedded trapped modes: (c) NAA at $l / h=2.999, K / 2 \pi=0.527$, (d) NSA at $l / h=3.676, K / 2 \pi=0.569$, (e) $\mathrm{NAA}$ at $l / h=1.759, K / 2 \pi=0.872$, (f) NSA at $l / h=1.214, K / 2 \pi=0.840$.

metric about the duct centre line. The symmetric problem is of considerable importance for wind tunnel testing of cavities and does not have a non-embedded trapped mode domain like the antisymmetric problem discussed above. Koch (2004) (no flow) as well as Alvarez \& Kerschen (2005) (with flow) pointed out significant differences in the performance of rectangular cavities in free space and in a duct which explain experimentally observed discrepancies, cf. Cattafesta III et al. (1997). The reason for these differences is the existence of trapped or almost trapped modes in a ducted domain (for the case with flow one would expect only almost trapped modes, cf. the corresponding investigation of Koch (1983) or Duan (2004) for the similar Parker mode problem). In order to allow a comparison with the free space cavity resonances Koch (2005) used the cavity length $l$ as reference length. However, it is exactly the cavity length which governs the occurrence of embedded trapped modes. Therefore, as in the antisymmetric problem, we use the duct height $h$ as reference length in this investigation to allow a direct comparison with the embedded trapped modes of Duan (2004). For our comparison we chose the same cavity parameters as for the antisymmetric example, namely $d / h=1$, but now with Neumann 
boundary conditions on the duct centre line. The values of the first six embedded modes which are symmetric in $x$ (NSS) and those which are antisymmetric in $x$ (NAS), are given in table 1, and a comparison is made with the complex resonances of Koch (2005) in figure 12. As can be seen the embedded modes correspond well with the values of the complex resonances at which there is zero damping. Figure 13 shows eigenfunctions $\operatorname{Re}(\phi)$ for selected symmetric embedded trapped cavity modes.

Figures 14 and 15 illustrate the variation of the aspect ratio of a cavity $l / 2 b$ which supports NSS embedded trapped modes with cavity depth. As $b / h \rightarrow \infty$, the value of $l / 2 b$ for each branch of modes tends to one of the values associated with the double eigenvalues of the enclosed rectangular cavity, given by (2.35),

$$
\frac{l}{2 b}=\left[\frac{n^{2}-p^{2}}{q^{2}-m^{2}}\right]^{1 / 2}, \quad m, n, p, q \text { integer, } p<n, m<q,
$$

and the branch can be designated by the four integers $(n, m, p, q)$. The branches in figure 14 all have $m=0$ and correspond to the side condition $K l=2 n \pi$, whereas the modes in figure 15 correspond to the alternative side condition in (2.21) $U_{0}^{I}=0$. As $K h<2 \pi$,

$$
\frac{l}{2 b}=\left(\frac{h}{2 b}\right) \frac{K l}{K h}>\left(\frac{h}{2 b}\right) \frac{K l}{2 \pi},
$$

and so if $K l=2 n \pi$,

$$
\frac{l}{2 b}>n\left(\frac{h}{2 b}\right),
$$

and the branches of modes lie above the curves $l / 2 b=n h / 2 b$, as shown in figure 14 . The value of $K h$ at the intersection point of these curves and the mode branches is the cut-off value $K h=2 \pi$, and $K h \rightarrow 0$ as the cavity depth, $2 b / h$ increases along each branch. In figure 15 , the mode frequencies asymptotically satisfy

$$
K l=\pi\left[4 n^{2}+\frac{m^{2} l^{2}}{b^{2}}\right]^{1 / 2},
$$

as given in (2.36). There is no obvious reason why the branches of modes should emanate from the curves given by the limiting equality obtained by substituting (6.4) into (6.2), namely

$$
\frac{l}{2 b}=\frac{h}{2 b} \frac{1}{2}\left[4 n^{2}+\frac{m^{2} l^{2}}{b^{2}}\right]^{1 / 2} .
$$

However the numerical evidence is that they do, albeit not always from above the curve, with the value of $K h$ given by $K h=2 \pi$ at the intersection point, and with $K h$ decreasing along the branch.

Similar branch diagrams may be obtained for modes with other symmetries about the $x$ - and $y$ - axis of the guide.

\section{Resonances in a ball-type valve model}

In this last section we compute the resonances in a two-dimensional model of a ball-type valve in an infinite duct as sketched in figure 1e. Neglecting the small gap between the valve body and the valve housing we have essentially non-symmetrical wall protrusions and wall cavities which vary with valve angle $\alpha$. The real and imaginary part of the lowest four complex resonances are depicted in figure 16 as function of valve angle $\alpha$.

If the valve is completely closed at $\alpha=90^{\circ}$ a finite size enclosed cavity is formed by the 

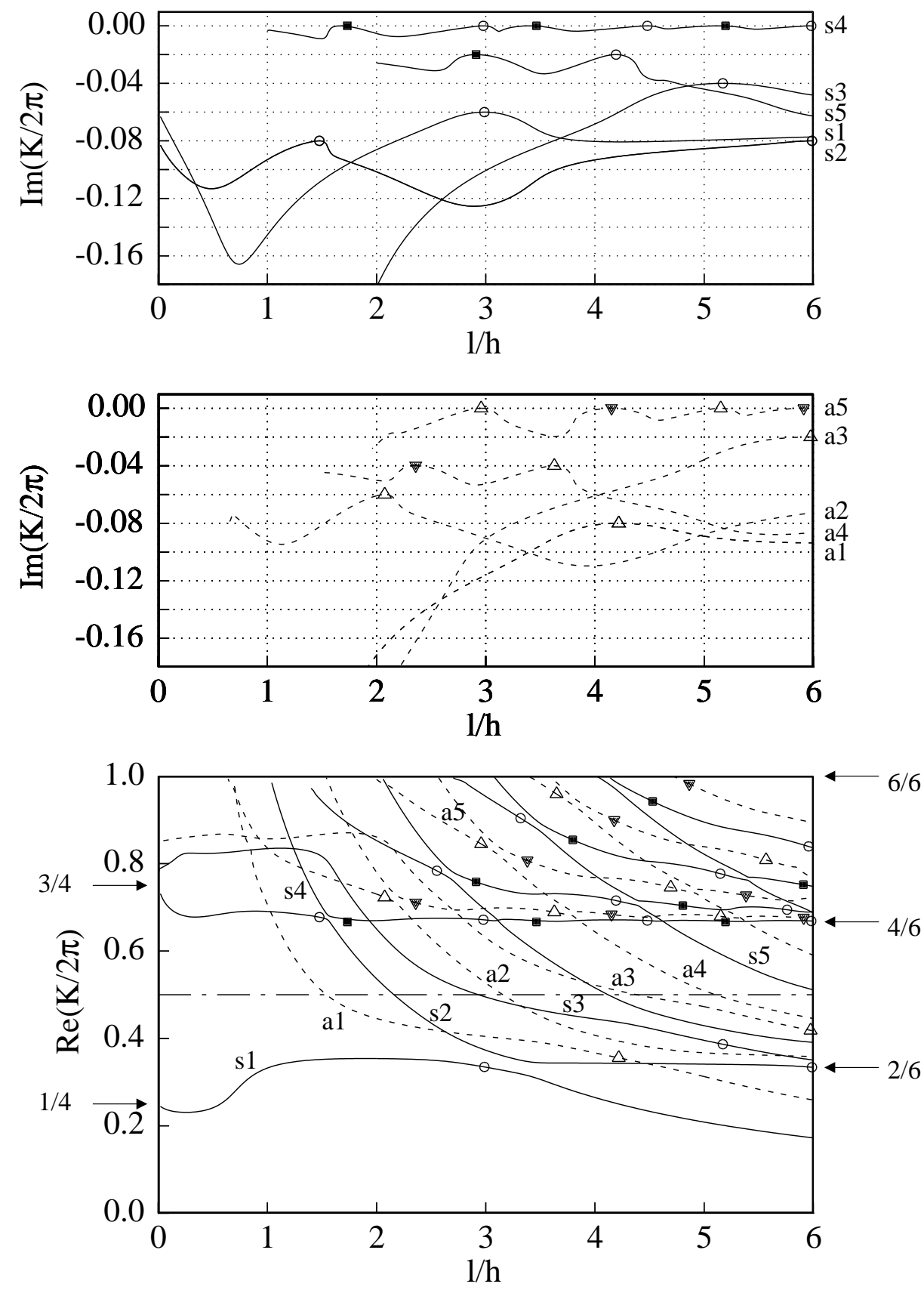

FIGURE 12. NSS and NAS resonances and embedded trapped modes for rectangular cavity in duct with $d / h=1$ : (a) Variation of resonant frequency $\operatorname{Re}(K / 2 \pi)$ with $l / h$. (b) Variation of resonance damping $\operatorname{Im}(K / 2 \pi)$ of NAS modes with $l / h$. (c) Variation of resonance damping $\operatorname{Im}(K / 2 \pi)$ of NSS modes with $l / h$. (To distinguish the individual damping curves the origin of the ordinate of each curve is shifted by -0.02). ०, • embedded trapped NSS modes; $\triangle, \nabla$ embedded trapped NAS modes. 
(e)

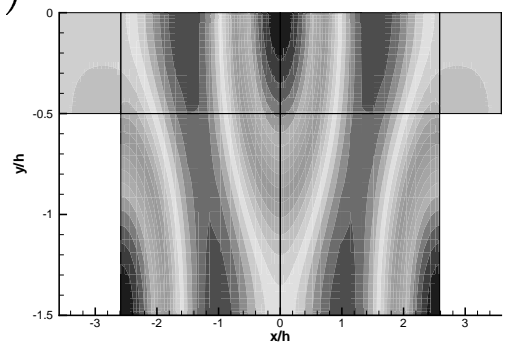

(c)

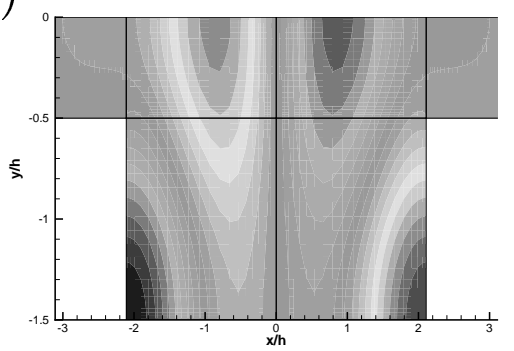

(a)

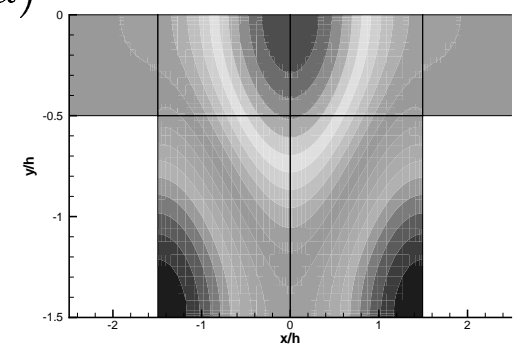

(f)

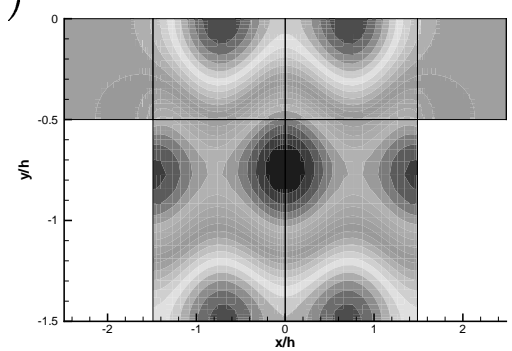

(d)

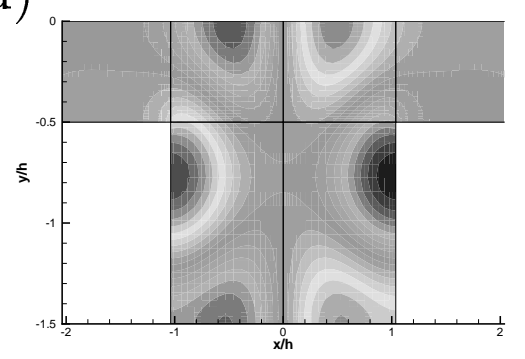

(b)

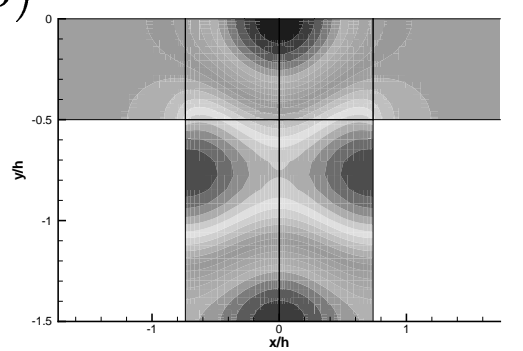

FiguRE 13. Selected eigenfunctions $\operatorname{Re}(\phi)$ of symmetric embedded trapped modes: (a) $\mathrm{NSS}$ at $l / h=2.988, K / 2 \pi=0.335$, (b) NSS at $l / h=1.476, K / 2 \pi=0.678$, (c) NAS at $l / h=4.218, K / 2 \pi=0.356$, (d) NAS at $l / h=2.074, K / 2 \pi=0.723$, (e) NSS at $l / h=5.171, K / 2 \pi=0.387$, (f) NSS at $l / h=2.978, K / 2 \pi=0.672$.

valve with four symmetries $S S, S A, A S$ and $A A$, where according to our nomenclature the first letter denotes the symmetry in $x$ and the second letter the symmetry in $y$. If the resonances are computed numerically for the enclosed cavity, i.e. without PML, we obtain the real resonances marked by the arrows on the right-hand side of figure 16a. The two modes which are symmetric in $y$ quickly reach high damping if the valve is opened and our numerical method becomes inaccurate. On the other hand the mode starting out with SA symmetry is only weakly damped and approaches the first antisymmetric duct cut-on mode with zero damping as $\alpha \rightarrow 0$. Near this cut-on frequency the wave moves almost tangentially along the PML and we had to increase the depth of the PML from $d_{P M L}=1.5$ to $d_{P M L}=6$ in order to obtain the results for the imaginary part of the SA resonance in figure $16 \mathrm{~b}$. As can be seen, the imaginary part in figure $16 \mathrm{~b}$ still is not quite zero. Near $\alpha=90^{\circ}$ we had to refine the grid near the singular corners to approach the limiting real resonances. Apparently the solution exhibits a boundary-layer behaviour because nearby values did not change with grid refinement. Of particular interest is the resonance starting out with AA symmetry because the imaginary part seems to approach 


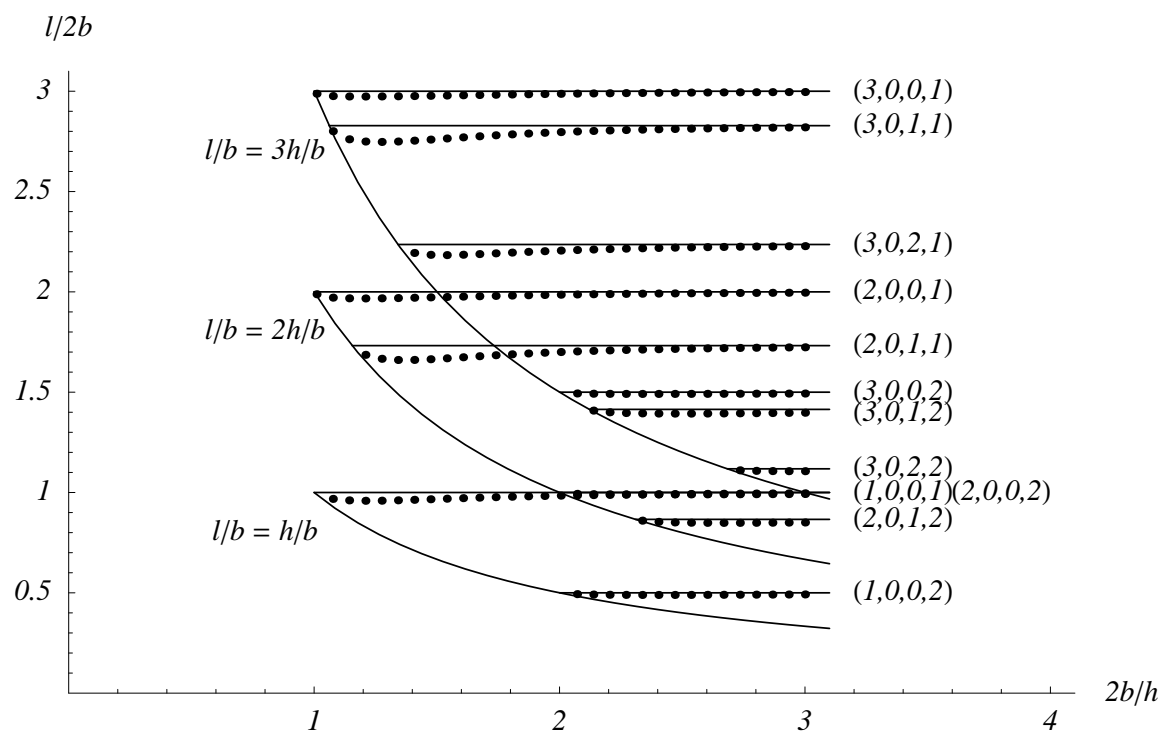

FIGURE 14. Variation of aspect ratio of cavity with cavity depth for which NSS embedded trapped modes $(\bullet)$ exist with side condition $K l=2 n \pi$.

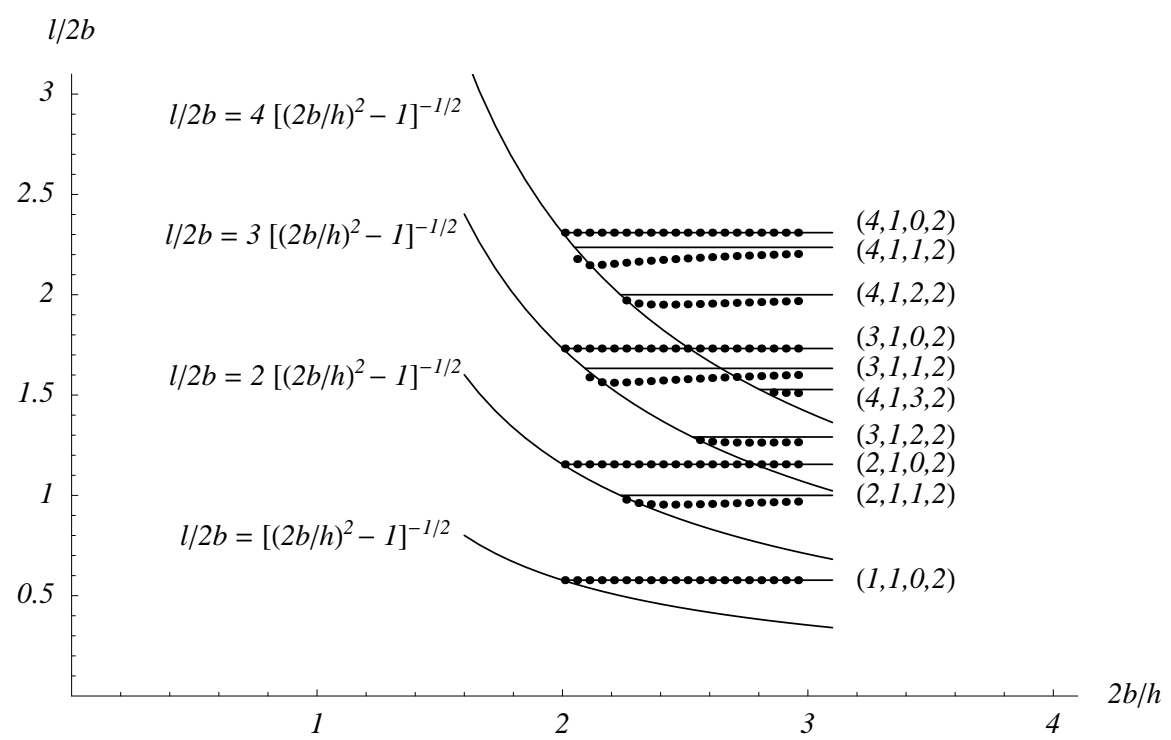

FigURE 15. Variation of aspect ratio of cavity with cavity depth for which NSS embedded trapped modes $(\bullet)$ exist with side condition $U_{0}^{I}=0$.

zero near $\alpha=42^{\circ}$. Whereas the AA symmetry is lost away from $\alpha=90^{\circ}$ there is still rotational symmetry about the origin, i.e. $\phi(-x,-y)=\phi(x, y)$. Our numerical method does not allow any conclusion whether this is a genuine trapped mode or only a neartrapped mode. But even if it is only a near-trapped mode one would expect $\alpha \approx 42^{\circ}$ to be the noisiest valve position because this resonance could be excited most easily by shear layer instabilities. 

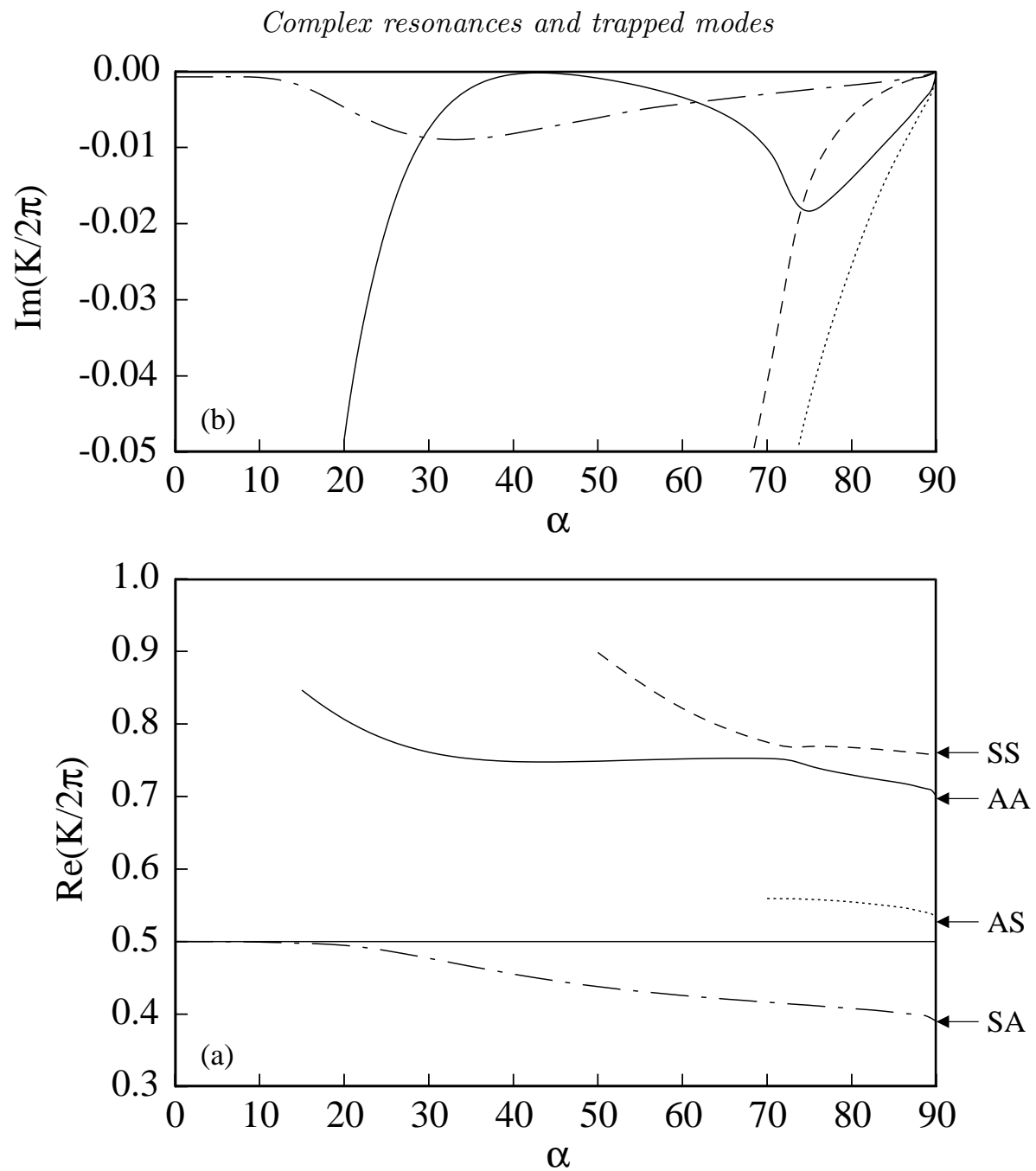

FigURE 16. Resonances of ball-type valve in a duct as function of valve angle $\alpha$ : (a) real part and (b) imaginary part of four lowest order complex resonances with $\sigma_{0}=1$.

\section{Conclusions}

In this work we have calculated embedded trapped mode frequencies for a variety of configurations of waveguides which contain structures, and compared them with numerically obtained complex resonances. In particular, we have shown that the modes which are known to exist for plates, circles and ellipses in guides correspond exactly to complex resonances with vanishingly small imaginary part. Furthermore, new embedded modes have been found for rectangular cavities with specific aspect ratios, placed symmetrically either side of a duct.

When the system is symmetric about the duct axis, trapped modes are known to exist at discrete frequencies below the first duct cut-off frequency, for almost all geometries. However embedded trapped modes exist only at isolated frequencies above the first cutoff for particular geometries. Indeed all the results obtained in this work, and other papers, suggest that in order for an embedded trapped mode to exist for a structure in a duct and within a particular class, an extra geometrical parameter is required, for each additional propagating duct mode, to force its amplitude to be zero. If there was no 
additional parameter, it was still sometimes possible to find near-trapped modes with very low damping, by exploiting the structure of the streamlines and pressure contours of a genuine, lower frequency, embedded mode. These complex resonances were computed by our numerical method by varying relevant parameters and searching for minima in the damping. Examples are the computed resonances for a circular cylinder in a guide above the second cut-off frequency and the one found in the ball-type valve model. Both the trapped modes and the near-trapped modes with low damping are of great importance in applications because at the corresponding frequencies, the system can be excited very easily by external forcing, such as vortex shedding when the structure is in a cross-flow.

\section{References}

Aguilar, J. \& Combes, J. 1971 A class of analytic perturbations for one-body Schrödinger Hamiltonians. Commun.Math.Phys. 22, 269-279.

Alvarez, J. \& Kerschen, E. 2005 Influence of wind tunnel walls on cavity acoustic resonances. AIAA-Paper 2005-2804. 11th AIAA/CEAS Aeroacoustics Conference, Monterey, California.

Aslanyan, A., Parnovski, L. \& Vassiliev, D. 2000 Complex resonances in acoustic waveguides. Q.Jl Mech.Appl.Math. 53, 429-447.

BASlev, E. \& Combes, J. 1971 Spectral properties of many body Schrödinger operators with dilation analytic interactions. Commun.Math.Phys. 22, 280-294.

BÉREnger, J. 1994 A perfectly matched layer for the absorption of electromagnetic waves. J.Comput.Phys. 114, 185-200.

Callan, M., Linton, C. \& Evans, D. 1991 Trapped modes in two-dimensional waveguides. J.Fluid Mech. 229, 51-64.

Cattafesta III, L., Garg, S., Choudhari, M. \& Li, F. 1997 Active control of flowinduced cavity resonance. AIAA-Paper 97-1804. 28th AIAA Fluid Dynamics Conference, Snowmass Village, CO.

Chew, W., Jin, J. \& Michielssen, E. 1997 Complex coordinate stretching as a generalized absorbing boundary condition. Microwave Optical Technol.Lett. 15 (3), 144-147.

Chew, W. \& Weedon, W. 1994 A 3-D perfectly matched medium from modified Maxwell's equation with stretched coordinates. Microwave Optical Technol.Lett. 7 (13), 599-604.

Collino, F. \& Monk, P. 1998 The perfectly matched layer in curvilinear coordinates. SIAM J.Sci.Comput. 19 (6), 2061-2090.

Davies, E. \& Parnovski, L. 1998 Trapped modes in acoustic waveguides. Q.Jl Mech.Appl.Math 51, 477-492.

Dequand, S., Hulshoff, S. \& Hirschberg, A. 2003 Self-sustained oscillations in a closed side branch system. J.Sound Vibration 265 (2), 359-386.

DuAn, Y. 2004 Trapped modes and acoustic resonances. PhD thesis, Loughborough University.

DuAn, Y. \& McIver, M. 2002 Embedded trapped modes near an indentation in an open channel. In 17th International Workshop on Water Waves and Floating Bodies (ed. R.C.T.Rainey \& S.F.Lee), pp. 37-40. Peterhouse, Cambridge, UK.

EAST, L. 1966 Aerodynamically induced resonance in rectangular cavities. J.Sound Vibration 3 (3), 277-287.

Evans, D. \& Fernyhough, M. 1995 Edge waves along periodic coastlines. Part 2. J.Fluid Mech. 297, 307-325.

Evans, D., Levitin, M. \& Vassiliev, D. 1994 Existence theorems for trapped modes. J.Fluid Mech. 261, 21-31. 
Evans, D. \& Linton, C. 1991 Trapped modes in open channels. J.Fluid Mech. 225, $153-175$.

Evans, D. \& Linton, C. 1994 Acoustic resonance in ducts. J.Sound Vibr. 173, 85-94.

Evans, D., Linton, C. \& Ursell, F. 1993 Trapped mode frequencies embedded in the continuous spectrum. Q.Jl Mech.Appl.Math. 46, 253-274.

Evans, D. \& Porter, R. 1997 Trapped modes about multiple cylinders in a channel. J.Fluid Mech. 339, 331-356.

Evans, D. \& Porter, R. 1998 Trapped modes embedded in the continuous spectrum. Q.Jl Mech.Appl.Math 52, 263-274.

Exner, P., ŠEBA, P., TAter, M. \& VAnĚK, D. 1996 Bound states and scattering in quantum waveguides coupled laterally through a boundary window. J.Math.Phys. 37, $4867-4887$.

Franklin, R. 1972 Acoustic resonance in cascades. J.Sound Vibration 25, 587-595.

Grikurov, V. 2004 Scattering, trapped modes and guided waves in waveguides and diffraction gratings. In Proceedings of the First East-West Workshop on Advanced Techniques in Electromagnetics. Warsaw, May 20-21, 2004 (arXiv:quant-ph/0406019).

Groves, M. 1998 Examples of embedded eigenvalues for problems in acoustic waveguides. Math.Methods in the Appl.Sci. 21 (6), 479-488.

Hein, S., Hohage, T. \& KoCh, W. 2004 On resonances in open systems. J.Fluid Mech. 506, 255-284.

Hein, S., KосH, W. \& SchöBerL, J. 2005 Acoustic resonances in a 2d high lift configuration and a 3 d open cavity. AIAA-Paper 2005-2867. 11th AIAA/CEAS Aeroacoustics Conference, Monterey, California.

Koch, W. 1983 Resonant acoustic frequencies of flat plate cascades. J.Sound Vibration 88, 233-242.

KocH, W. 2004 Acoustic resonances in rectangular open cavities. AIAA-Paper 20042843. 10th AIAA/CEAS Aeroacoustics Conference, Manchester, UK.

Koch, W. 2005 Acoustic resonances in rectangular open cavities. AIAA Journal 43 (11), $2342-2349$

Kriesels, P., Peters, M., Hirschberg, A., Wijnands, A., Iafrati, A., RiCCARdi, G., Piva, R. \& Bruggeman, J. 1995 High amplitude vortex-induced pulsations in a gas transport system. J.Sound Vibration 184 (2), 343-368.

Linton, C. \& Evans, D. 1992 Integral equations for a class of problems concerning obstacles in waveguides. J.Fluid Mech. 245, 349-365.

Linton, C. \& McIver, M. 2002 The existence of Rayleigh-Bloch surface waves. J.Fluid Mech. 470, 85-90.

Linton, C., McIver, M., McIver, P., Ratcliffe, K. \& Zhang, J. 2002 Trapped modes for off-centre structures in guides. Wave Motion 36, 67-85.

Linton, C. \& Ratcliffe, K. 2004 Bound states in coupled guides. I.Two dimensions. J.Math.Physics 45 (4), 1359-1379.

MANIAR, H. \& NEWMAN, J. 1997 Wave diffraction by a long array of cylinders. J.Fluid Mech. 339, 309-330.

McIver, M., Linton, C., McIver, P., Zhang, J. \& Porter, R. 2001 Embedded trapped modes for obstacles in two-dimensional waveguides. Q.Jl Mech.Appl.Math 54 (2), 273-293.

McIver, M., Linton, C. \& Zhang, J. 2002 Branch structure of embedded trapped modes in two-dimensional waveguides. Q.Jl Mech.Appl.Math 55, 313-326.

Moiseyev, N. 1998 Quantum theory of resonances: calculating energies, widths and cross-sections by complex scaling. Physics Reports 302, 211-293.

NAyfeh, A. \& Huddleston, D. 1979 Resonant acoustic frequencies of parallel plates. 
AIAA-Paper 79-1522. 12th AIAA Fluid and Plasma Dynamics Conference, Williamsburg, Virginia.

PARKER, R. 1966 Resonance effects in wake shedding from parallel plates: Some experimental observations. J.Sound Vibration 4 (1), 62-72.

PARKER, R. 1967 Resonance effects in wake shedding from parallel plates: Calculation of resonant frequencies. J.Sound Vibration 5, 330-343.

Parker, R. \& Stoneman, S. 1989 The excitation and consequences of acoustic resonances in enclosed fluid flow around solid bodies. Proc.Instn.Mech.Engrs. 203, 9-19.

Porter, R. \& Evans, D. 1999 Rayleigh-Bloch surface waves along periodic gratings and their connection with trapped modes in waveguides. J.Fluid Mech. 386, 233-258.

Porter, R. \& Evans, D. 2005 Embedded Rayleigh-Bloch surface waves along periodic rectangular arrays. Wave Motion 43, 29-50.

Reethof, G. 1978 Turbulence-generated noise in pipe flow. Ann.Rev.Fluid Mech. 10, 333-367.

SCHÖBERL, J. 1997 NETGEN An advancing front 2D/3D-mesh generator based on abstract rules. Computing and Visualization in Science 1, 41-52.

Simon, B. 1973 The theory of resonances for dilation analytic potentials and the foundations of time dependent perturbation theory. Ann.Math. 97, 247-274.

Sugimoto, N. \& Imahori, H. 2006 Localized mode of sound in a waveguide with Helmholtz resonantors. J.Fluid Mech. 546, 89-111.

Ursell, F. 1951 Trapping modes in the theory of surface waves. Proc.Camb.Phil.Soc. 47, 347-358.

UtsunomiYa, T. \& TAYlOR, R. 1999 Trapped modes around a row of circular cylinders in a channel. J.Fluid Mech. 386, 259-279.

Woodley, B. \& Peake, N. 1999 Resonant acoustic frequencies of a tandem cascade. Part 1: Zero relative motion. J.Fluid Mech. 393, 215-240.

ZiadA, S. \& Shine, S. 1999 Strouhal numbers of flow-excited acoustic resonance of closed side branches. J.Fluids Struct. 13 (1), 127-142. 